\title{
A comprehensive approach for artifact-free sample preparation and assessment of mitochondrial morphology in tissue and cultured cells
}

Antentor Hinton, Jr. ${ }^{1,2,3 *}$, Prasanna Katti ${ }^{5 *}$, Trace A. Christensen ${ }^{3 *}$, Margaret Mungai ${ }^{1,2}$, Jianqiang Shao $^{6}$, Liang Zhang ${ }^{7}$, Sergey Trushin ${ }^{7}$, Ahmad Alghanem ${ }^{8,9}$, Adam Jaspersen ${ }^{3}$, Rachel E. Geroux ${ }^{7}$, Kit Neikirk $^{11}$, Michelle Biete ${ }^{11}$, Edgar Garza Lopez ${ }^{4}$, Zer Vue ${ }^{4}$, Heather Beasley ${ }^{4}$, Andrea Marshall ${ }^{4}$, Innes Hicsasmaz ${ }^{1,2}$, Ranthony A.C. Edmonds ${ }^{10}$, Andres Dajles ${ }^{1}$, Young Do Koo ${ }^{1,2}$, Serif Bacevac ${ }^{1,2}$, Jeffrey L. Salisbury $^{3,12}$, Renata O. Pereira ${ }^{1,2}$, Brian Glancy ${ }^{5,13}$, Eugenia Trushina ${ }^{7,14 \#}$, E. Dale Abel ${ }^{1, \#}$

1. Department of Internal Medicine, University of Iowa - Carver College of Medicine, Iowa City, IA.

2. Fraternal Order of Eagles Diabetes Research Center, Iowa City, IA.

3. Microscopy and Cell Analysis Core Facility, Mayo Clinic, Rochester, MN

4. Department of Molecular Physiology and Biophysics, Vanderbilt University, Nashville, TN

5. National Heart, Lung, and Blood Institute National Institutes of Health, Bethesda, MD

6. Central Microscopy Research Facility, Iowa City, IA

7. Department of Neurology, Mayo Clinic, Rochester, MN

8. Department of Internal Medicine, Division of Cardiology, Washington University in St. Louis, St. Louis, MO

9. King Abdullah International Medical Research Center (KAIMRC), Riyadh, Saudi Arabia

10. Department of Mathematics, Ohio State University, Columbus, $\mathrm{OH}$

11. College of Natural and Health Sciences, University of Hawaii at Hilo, Hilo, HI

12. Department of Biochemistry and Molecular Biology, Mayo Clinic, Rochester, MN

13. National Institute of Arthritis and Musculoskeletal and Skin Diseases, National Institutes of Health, Bethesda, MD

14. Department of Pharmacology and Experimental Therapeutics, Mayo Clinic, Rochester, MN 
* Authors Contributed Equally to Manuscript

\# Co-Senior Authors

\# Co-corresponding authors

E. Dale Abel, M.D., Ph.D.

Fraternal Order of Eagles Diabetes Research Center

Carver College of Medicine,

University of Iowa,

Iowa City, IA, USA 52242

Phone: 319-356-4379

DRCAdmin@uiowa.edu

Eugenia Trushina, Ph.D.

200 First Street SW,

Guggenheim Building

Rochester, MN 55905

Phone: 507-284-8197

trushina.eugenia@mayo.edu 


\begin{abstract}
Mitochondrial dynamics and morphology (fission, fusion, and the formation of nanotunnels) are very sensitive to the cellular environment and may be adversely affected by oxidative stress, changes in calcium levels, and hypoxia. Investigating the precise relationship between the organelle structure and function requires methods that can adequately preserve the structure while providing accurate, quantitative measurements of mitochondrial morphological attributes. Here, we demonstrate a practical approach for preserving and measuring fine structural changes in two-dimensional electron micrographs, obtained using transmission electron microscopy, highlighting the specific advantages of this technique. Additionally, this study defines a set of quantifiable metrics that can be applied to measure mitochondrial architecture and other organellar structures. Finally, we validated specimen preparation methods that avoid the introduction of morphological artifacts in mitochondrial appearance that do not require wholeanimal perfusion.
\end{abstract}

\title{
Introduction
}

Mitochondria are responsible for meeting the energetic and metabolic demands of all cells and are involved in critical signaling pathways. Importantly, the balance between health and disease rests, in part, on environmental, genetic, and biochemical factors that influence mitochondrial function. Dynamic transitions in mitochondrial characteristics can reflect normal and disease-related changes in mitochondrial size and shape, changes in network structures, and altered interactions with other organelles ${ }^{1-11}$ 1-11 $^{\text {. Mitochondrial number and shape are determined, in part, by fusion and fission cycles }}{ }^{1-}$ ${ }^{61-6}$, which are coordinated by the fusion genes, optic atrophy 1 (OPA-1) and mitofusin 1 and 2 (MFN-1 and MFN2, respectively), the fission protein dynamin-related protein 1 (DRP-1) and its receptors, mitochondrial fission 1 (FIS1), mitochondrial fission factor (MFF), and mitochondrial dynamics protein MiD49 and MiD51 $1^{1-6}$ 1-6. 
Mitochondria interact with other intracellular organelles, including the endoplasmic reticulum (ER), through direct mitochondria-ER ${ }^{3,6,12-18}$ contacts (MERCs), and lipid droplets (LDs), which can occur through transient, kiss-and-run contacts or the longer-lived LD-anchored mitochondria (LDAM) ${ }^{1}$. Additionally, mitochondria also interact with the nucleus via the release of metabolic intermediates that mediate transcriptional regulation ${ }^{3,6,12-18}$. Furthermore, mitochondria form dynamic branching networks and connections by forming nanotunnels, or mitochondria-on-a-string (MOAS) ${ }^{9,19-22}$, that link mitochondrial elements to one another in response to energetic stress, hypoxia, and other physiological or environmental changes. These nanotunnels and MOAS are often found in different disease states, including Alzheimer's Disease ${ }^{4,19-22}$, and their formation is thought to represent a mechanism for promoting mitochondrial communication and protecting mitochondria from fragmentation and degradation by lysosomes ${ }^{4,20-23}$. These unique structures are formed within few minutes under hypoxic conditions. Since methods employed for specimen preparation for electron microscopy could also induce hypoxia in cells and tissues, it is imperative to develop methodology that will produce artifact-free specimens allowing for reliable and reproducible assessment of mitochondrial morphology. However, since the original independent discovery by both Palade and Sjostrand in $1953^{23,24}$, a myriad of modifications of the fixation, dehydration, sectioning, and staining of tissue sections have been developed to better preserve the native mitochondrial morphology ${ }^{25-33}$. Yet, there is not a clear consensus on what EM technique resolves mitochondria the best.

Notably, earlier studies of mitochondrial structure have used various types of fixation methods; however, these methods have not resulted in a consistent way to access ultrastructure. For example, the mitochondria are very sensitive to fixation artifacts; many fixation methods induce fixation artifacts, as verified by light microscopy ${ }^{34,35}$. This leads us to question what fixation methods best preserve mitochondria for TEM and similar applications, such as 3D reconstruction. Although there are many methods available for studying organelle structural features and identifying dynamic changes ${ }^{4-9}$, a standardized method enables the rigorous comparison of findings between diverse groups remains elusive 
for electron microscopy-based applications ${ }^{25,28}$. In addition, the novel MOAS or nanotunnel phenotype was only recently discovered ${ }^{4,37-42}$. Previously, no systematic approach had been defined to characterize mitochondrial morphology in 3D or identify MOAS in multiple tissue types. Thus, it is essential to methodically define mitochondrial architecture, which may reveal mechanistic features in clinical or experimental samples. Novel unidentified structures will be uncovered as the EM reconstruction technology matures and pragmatic approaches are developed.

The objectives of this study were to 1) develop an optimized approach for specimen handling and fixation to preserve mitochondrial morphology, 2) optimize methodology for quantifying organelle characteristics using transmission electron microscopy (TEM) micrographs, and 3) validate the reproducibility of these methods for describing morphological changes in subcellular organelles. We focused on methods for quantifying indices of mitochondrial morphology ${ }^{1-6}$, such as hyperbranching, mitochondrial volume, cristae morphology $\mathrm{y}^{7-11}$, as well as the length and percentage coverage of MERCs ${ }^{12-16}$. To further validate our approach, we deleted genes encoding known mitochondrial dynamics proteins, such as OPA-1, MFN2, and DRP-1, to evaluate which methodology was the most reproducible and representative of mitochondrial changes.

To achieve this goal, we used widely available open-source image analysis platforms, such as ImageJ (FIJI), proprietary software, and image analysis tools to refine approaches for structural quantification using EM micrographs. We present a comprehensive overview of the methods that could be used to standardize the quantification of mitochondrial morphology. Finally, we optimize conditions that ensure the generation of the artifact-free samples in cells and tissue, to assess mitochondrial morphology with EM reconstruction using a manual serial-section TEM.

\section{$\underline{\text { Results }}$}




\section{Artifact-free Tissue Preparation for Ultrastructural Studies Using EM}

The techniques commonly used to collect tissue for EM analysis include multiple modes of anesthesia and cardiac perfusion with pre-flush with phosphate-buffered saline (PBS) solution to remove erythrocytes from blood vessels. Common anesthetics include ketamine/xylazine injection, $\mathrm{CO}_{2}$ inhalation, or 5\% isoflurane/oxygen mixture inhalation. Cardiac perfusion ensures thorough fixation of the whole body for the analysis of multiple tissues. However, this method of fixation prohibits the performance of any other assays, such as Western blot or RNA sequencing, that require fresh tissue. In contrast, other methods of euthanasia, such as $\mathrm{CO}_{2}$ or isoflurane inhalation, with subsequent cervical dislocation allow the collection of multiple tissues for various analyses from the same animal, making these approaches attractive. To determine whether these anesthetic agents could induce hypoxic conditions, we first compared mitochondrial morphology in hippocampal brain tissue of wild-type (WT) mice anesthetized with $\mathrm{CO}_{2}(3 \mathrm{~min})$ or $5 \%$ isoflurane/oxygen before cervical dislocation (Fig. 1A-C). The whole brain was quickly removed and sliced; hippocampal tissue (3 mm $\times 3 \mathrm{~mm} \times 1$-mm thickness) was dissected and immediately immersed in Trump's solution for fixation

(Fig. 1A). Consecutive serial sections of tissues subjected to each of the anesthetic techniques were examined using TEM. We found that $\mathrm{CO}_{2}$ inhalation induced a prominent MOAS phenotype (Fig. 1B), whereas the tissue prepared from mice anesthetized using a mixture of 5\% isoflurane/oxygen did not exhibit significant MOAS formation (Fig. 1C) or changes in MERC formation (Figure S2D); however, 5\% isoflurane/oxygen demonstrated uniformly elongated mitochondria (Fig. 1C).

Next, we examined the effect of different methods of transcardial perfusion on mitochondrial morphology in brain tissue (Fig. 1A,D,E). WT mice were anesthetized by ketamine/xylazine injection. Mice were perfused with either PBS followed with 4\% paraformaldehyde (PFA), or with 4\% PFA alone without a PBS flush (Fig. 1D). Although the PBS/PFA procedure typically requires approximately 5 minutes per mouse, the perfusion with PFA alone can be performed in less than 3 minutes. Intact brains were removed and post-fixed overnight at room temperature in Trump's solution. The hippocampal CA1 region and 
cortex were dissected from each brain and processed for TEM. The perfusion of animals with PBS before PFA fixation resulted in MOAS formation, not only in the hippocampi (Fig. 1C) but also in the cortex (data not shown). In contrast, animals perfused with PFA alone, without a prior PBS flush, had uniformly elongated mitochondria throughout different brain regions (Fig. 1F). These data suggest that transcardial perfusion with a PBS flush before fixation may create hypoxic conditions that affect mitochondrial morphology.

Simple immersion fixation of tissue is often performed instead of whole-body fixation using cardiac perfusion. The success of immersion fixation depends on how quickly the fixative could penetrate the immersed tissue. If the tissue is too large, slow fixation could reduce tissue oxygenation leading to hypoxia. To determine optimum conditions for immersion fixation, we compared two sets of brain tissue obtained from the same WT mouse (Fig. 1G). A mouse was sacrificed by cervical dislocation without prior anesthesia, the intact brain was rapidly removed (<2 $\mathrm{min})$. One hemisphere was sliced into 1-mmthick sections (Fig. 1, left), whereas the other hemisphere was cut in half (Fig. 1, right). All tissues were immediately immersed in Trump's fixative solution overnight. The hippocampal CA1 region and cortex were dissected from the 1-mm-thick tissue slices and the middle of each half of the other hemisphere (Fig. 1A) and then processed for TEM. Mitochondria from the 1-mm-thick slices were approximately $0.3 \mu \mathrm{m}$ in diameter and uniformly elongated in all brain regions examined (Fig. 1H, the hippocampus is shown). In contrast, mitochondria from the larger brain tissue samples exhibited a wide variety of shapes, including MOAS, in all regions examined (Fig. 1I, the hippocampus is shown). These data suggest that the immersion fixation of large pieces of tissue may lead to alterations in mitochondrial morphology due to slow penetration of the fixative leading to hypoxia-associated artifacts.

Taken together, our findings suggest that to preserve mitochondrial morphology in tissues for accurate and artifact-free examination using EM, suitable anesthetic agents include ketamine/xylazine or $5 \%$ isoflurane/oxygen, while $\mathrm{CO}_{2}$ must be avoided. If cardiac perfusion must be performed, avoid 
the PBS flush. For immersion fixation of fresh tissue, the size of the tissue should not exceed $1 \mathrm{~mm}$.

The proposed workflow for tissue preparation is presented in Fig. 1.

\section{Artifact-free Preparation for Ultrastructural Studies of Cultured Cells Using EM}

Next, we established optimal conditions to preserve the ultrastructure for EM examination of cultured cells. We examined the effects of different cell harvest and fixation methods on mitochondrial morphology (Figure 2A-H). The quality of TEM fixation allows the fine details of cristae morphology to be observed. To determine the optimal protocol for cultured cells, we compared eight fixation methods with differing scraping techniques and fixation times, then observed which method best preserved mitochondrial and cristae morphology (Figure 2A-H).

Insulin increases mitochondrial fusion in cultured cardiomyocytes and skeletal muscle cells ${ }^{1}$. Insulin stimulation results in larger, fused mitochondria with increased integrated cristae density ${ }^{1}$. Thus, using an insulin stimulation model permitted the validation of specimen preparation conditions under experimental conditions that increased mitochondrial size and cristae density. Before fixation, cells were treated with 2 hours of insulin stimulation ${ }^{1}$. The cultures were then prepared using a variety of harvesting and fixation methods to determine the optimal procedures for imaging.

\section{Scraping with Fixation}

We evaluated the quality of mitochondrial and cristae morphology in myotubes prepared from differentiated skeletal muscle satellite cells. Following insulin stimulation ( 2 hours of insulin treatment at $10 \mathrm{nmol} / \mathrm{L})^{1}$, treated myotubes were scraped and post-fixed for TEM (Figure $2 \mathrm{~A}$ ). We observed that scraping the cells before fixation altered the plasma membrane and damaged mitochondria. Similarly, following 2 hours of insulin treatment, scraping into TEM fixative (Figure 2B), scraping after fixation for 5 minutes (Figure 2C), and scraping after fixation for 10 minutes (Figure 2D) all resulted in poor cell 
preservation quality for TEM analysis (Figure 2A-D). Therefore, scraping cells damages the plasma membrane and mitochondrial integrity.

\section{Fixation with no Scraping}

To determine the optimal fixation procedures necessary to maintain mitochondrial integrity for TEM analysis, cultures were processed in 6-well plates (Figures 2E-H) and on coverslips (data not shown), and skeletal muscle cells were grown on Matrigel-coated plates (Figure 2E-H). Cultures at $80 \%$ confluency were fixed in TEM buffer directly in the 6-well plates by placing the plate in the incubator for 5 minutes (Figure 2E) in 37ロ, 10 minutes (Figure 2F), 30 minutes to 1 hour, (Figure 2G), and 24 hours (Figure 2H). The results showed that 10-minute fixation improved mitochondrial morphology and cristae integrity (Figure 2F) while fixing for 30 minutes to $1 \mathrm{hr}$ (Figure 2G) or for 24 hours (Figure 2H) further improved image quality.

\section{Systematic Quantification of Mitochondrial Morphology and Interactions with Organelles}

Specific details of the methodology applied to describe and quantify mitochondrial morphology in TEM are presented in the online methods. Results using these methods are presented to describe changes in mitochondrial morphology in response to hormonal and genetic manipulations that would be predicted to alter mitochondrial morphology or the interactions between mitochondria and other organelles.

\section{Determining MERC Distance in MFN-2-Deficient Cells in Murine and Drosophila models}

While several studies have reported that the ablation of MFN-2, a mitochondrial outer membrane fusion protein, alters the MERC distance ${ }^{5,10,11}$, other studies have disputed this conclusion ${ }^{12}$. To optimize methodology for ultrastructural quantification, we ablated MFN-2 from fibroblast cells using Cre recombinase. After deleting MFN-2, the MERC distance increased compared with the control, indicating that MFN-2 alters MERC distance (Figure 3A and B). Additionally, we used the ER-mitochondria contact coefficient (ERMICC) to calculate the percentage of ER covered by mitochondria and the percent 
coverage of mitochondria by ER. The percent coverage for both ER and mitochondria increased in MFN2-knockout $(\mathrm{KO})$ cells compared with the control cells (Figure 3C and D) ${ }^{5}$. In primary myotube cells, we transiently ablated MFN-2 using the CRISPR-Cas9 system, which resulted in a similar increase in the MERC distance compared with the control (Figure 3E, E', and F). We also ablated the Drosophila homolog of MFN-2, MARF, in Drosophila melanogaster using the RNAi-Gal4 system to test whether this process was conserved in other model organisms. We observed an increase in the MERC distance following MARF knockdown (KD) (Figure 3G, G', and H).

\section{Quantification of Mitochondrial Size and Shape Following DRP-1 Knockdown in Skeletal Muscle}

\section{Myoblasts}

DRP-1, or dynamin-1-like protein, is a GTPase that regulates mitochondrial fission. A recent study describing skeletal muscle function following the loss of DRP-1 demonstrated an elongation of mitochondria in tissue ${ }^{6}$. To confirm these findings, we ablated DRP-1 in a skeletal muscle cell line, which resulted in excessive mitochondrial fusion or the lack of mitochondrial fission, causing the formation of hyperfused mitochondria (Figure 4A and B). Notably, the deletion of DRP-1 led to an increase in mitochondrial area and length, as well as a decrease in mitochondrial number and the circularity index, when compared with the WT (Figure 3C-F) ${ }^{6,13}$. Thus, the deletion of DRP-1 prevented mitochondrial fission, resulting in elongated and less circular mitochondria. Interestingly, ER area was also increased following DRP-1 knockdown (Figure S6C, S6F).

\section{Mitochondrial Size and Cristae Morphology Following Insulin Treatment of Primary Human}

\section{Myoblasts}

Parra et al. demonstrated that insulin stimulation induces the transcription of OPA-1 in human myoblasts, leading to increased mitochondrial size ${ }^{4}$. We recapitulated these findings using human myoblast cells ${ }^{4}$ that underwent insulin stimulation and observed an increase in the mitochondria area. Treatment of 
primary human skeletal myoblasts with insulin $(10 \mathrm{nmol} / \mathrm{L} \text { for } 2 \text { hours })^{4}$ induced the appearance of hyperfused mitochondria and increased the number of cristae (Figure $4 G$ and $\left.4 G^{\prime}\right)^{9,17}$, particularly in the perinuclear and central areas (Figure $4 \mathrm{G}$ and $4 \mathrm{G}^{\prime}$ ). In addition, 2 hours of insulin stimulation increased the mitochondrial area (Figure 4H). Furthermore, we observed changes in the cristae morphology in response to insulin stimulation; specifically, a trending increase in the cristae number, cristae surface area, and cristate volume density, but with no change in cristae score (Figure 4I-L). Therefore, measuring only the cristae score is insufficient to assess cristae morphology, whereas quantifying the cristae number, cristae surface area, and cristae volume density represents a more thorough characterization of the cristae structural features. These data also suggested that insulin stimulation alters cristae morphology and mitochondrial size. Quantification demonstrated that insulin stimulation in human skeletal muscle cells increased mitochondrial fusion and increased the joining of cristae membranes (Figure 4A-L). Thus, our methodology confirmed previous observations linking insulin signaling with the regulation of mitochondrial morphology ${ }^{4}$.

\section{Measuring Changes in Cristae Morphology Following OPA-1 Ablation}

OPA-1, a mitochondrial GTPase protein, maintains cristae morphology and is responsible for inner mitochondrial fusion ${ }^{4}$. The loss of OPA-1 in myoblast cells decreased mitochondria area (Figures $4 \mathrm{~N}$ and 4M and 4M'). Additionally, OPA-1-deficient skeletal muscle cells exhibited decreased cristae numbers (Figure 4M, 4M', and 4O-R). The quantification of cristae morphology demonstrated a reduced cristae score, cristae number, cristae volume, and cristae surface area in OPA-1-deficient skeletal muscle cells compared with the control cells (Figure 4O-R). Furthermore, OPA-1-deficient cells also had increased tubular cristae (Figure 4S) and decreased lamellar cristae (Figure 4S) compared with the control cells.

\section{$\underline{\text { Discussion }}$}


With the development and availability of new techniques to assay mitochondrial structures in tissues, tissue preparation methods must be reliable and free of artifacts. We used serial-sectioning TEM reconstruction to develop a protocol that could be used to study mitochondrial morphology in various tissues, including the brain. We capitalized on our recent identification of a novel mitochondrial phenotype that forms in response to energetic stress and hypoxia. We also demonstrated that procedures that induce hypoxic conditions, including the use of certain anesthetics and perfusion methods, or delayed fixation, will likely introduce mitochondrial artifacts, such as MOAS. Moreover, our study demonstrated that the details of mitochondrial morphology in tissues could be more readily revealed using serialsectioning TEM reconstruction. We provide a comprehensive protocol for designing experiments to promote the most efficient use of animals and brain tissues. This protocol is suitable for conventional and advanced EM techniques using thick and thin tissue sections. In both cases, high-quality micrographs provide rich information, not only on mitochondrial structure but also on the other aspects of the cellular environment including the nucleus, the structure of nuclear pores, the mitochondria-ER network, the sizes and structures of synaptic boutons, and mitochondria found within neurites. The complexity of the intracellular environment can be examined in various tissues, including the brain, and is not limited to mitochondria. This protocol was tested using multiple sectioning techniques, making it suitable for a broad variety of EM reconstruction applications. Depending on the experimental design and the available sample group, the best tissue preparation techniques for EM examination in mice could be achieved through anesthetization by the inhalation of a mixture of $5 \%$ isoflurane/oxygen, followed by cervical dislocation (Figures 1,2). Using transcardial PBS perfusion before PFA delays fixation can result in artificial changes to mitochondrial morphology. For direct tissue fixation protocols, using smaller pieces of tissue rather than larger pieces can improve fixation. Adhering to these technical steps during the preparation of tissue intended for ultrastructural analyses will help avoid introducing artifacts and strengthen; in particular, observations regarding mitochondrial changes. These technical developments are important for understanding basic cell biology and the mechanisms that underlie diverse pathologies, including neurodegenerative diseases, cancer, diabetes, kidney and liver disease, and developmental 
disorders. In addition, these techniques can be applied to determine the best practices for imaging other tissues, such as skeletal muscle, from different model organisms, such as flies, cells, and mice (Figure 14).

\section{Measurements of Mitochondrial Cristae}

Multiple methods for analyzing cristae exist. The cristae score-a scoring system for cristae abundance and form ranging from 0 (worst) to 4 (best) - may be an appropriate measurement for use in tissue. However, the cristae score does not incorporate the actual total number of cristae for any measurement or point in time due to subjective judgement ${ }^{9}$. Thus, the most reliable measurements are cristae volume density, cristae surface area, and cristae number, which directly measure what happens to the cristae's folds or membrane after gene deletion or treatment ${ }^{4,8}$. Tissue mitochondria can be scored in a similar fashion using the actual mitochondrial volume density and cristae surface area. Furthermore, distinguishing which type of cristae are being altered by genes or treatment is also necessary. These findings can be scored using 2D TEM by examining changes in the typical morphology ${ }^{4,8,9}$.

\section{Measurement of MERCs}

MFN-2 deletion increases MERC distance and decreases the ERMICC ${ }^{5}$. Using our quantification methods, we were able to confirm these changes. We also used the percentage coverage of ER and mitochondria to measure the MERC space ${ }^{14}$. Conversely, we found that MFN-2 ablation increased ERmitochondria distance. ERMICC also has a greater percentage coverage of $\mathrm{MERCs}^{5}$, consistent with previously published studies ${ }^{14}$ that showed an increased MERC distance.

Furthermore, mitochondria adjust to the metabolic conditions through the parallel remodeling of the cristae and MERCs via a mechanism that degrades OPA-1 in an MFN-2-dependent pathway, suggesting that MERC distance can be altered depending on the energy conditions ${ }^{15}$. Additionally, Naon et al. showed that MFN-2 ablation led to a wider MERC distance ${ }^{5}$. Other studies revealed that loss of MFN-2 
increases MERC coverage using a different measurement involving the length of the ER-mitochondria contact normalized with either mitochondrial or ER surface area ${ }^{14}$. Here, we present a standardized and systematic method for measuring MERC and quantifying MERC distance, ERMICC, and percent coverage. This protocol can be used to further elucidate MERC dynamics and functions. For example, a smaller MERC coverage due to more considerable MERC distances suggests that the results are similar $^{16,17}$. However, increased MERC coverage associated with increased MERC distance may suggest a change in the $\mathrm{Ca}^{2+}$ level (the $\mathrm{Ca}^{2+}$ transfer effect) ${ }^{10}$. Additionally, smaller MERC distances can allow lipid $\operatorname{transfer}^{10}$. It is essential to understand specific MERC measurements and use all measurements when uncertain.

Moreover, the loss of MFN-2 can increase ER stress, which can result in ER ballooning due to an increase in eukaryotic translation initiation factor 2-alpha kinase (eIF2AK) and protein kinase R (PKR)-like ER kinase (PERK) activity, proteins involved in global protein translation attenuation and chaperone expression ${ }^{18}$. Additionally, the loss of the mitochondrial protein DRP-1, which travels from the cytosol to the mitochondria to promote fission, can induce ER stress ${ }^{6}$. This increase in ER ballooning can be measured using percentage coverage to determine the surface area of the ER or mitochondria associated with MERCs ${ }^{22,30}$. Following the deletion of DRP-1 in primary skeletal myoblasts, we identified an increase in the ER surface area (Figure S6 A-C). Additionally, the MFN-2 CRISPR-mediated KO had an increased ER surface area compared with the control cells (Figure S6 D-F).

\section{Limitations and Considerations:}

In this study, we demonstrated quantification methods that can be applied to the systematic analysis of organelles in tissues and cells (Figures 3-4 and S1-S8). Unless only a few mitochondria demonstrate overall changes, we suggest using a histogram to examine shifts in the distribution of the mitochondrial $\operatorname{area}^{4}$. Additionally, a histogram gives insight into mitochondrial size, which may be altered in response to 
gene deletion or treatment. For instance, when assessing the number of mitochondria per cell or total mitochondrial volume, we have encountered a limitation in the number of mitochondria in each plane.

Other tools besides the imaging techniques detailed above can be used to evaluate MERC tethering. To determine the tethering between two molecules, the changes in MERC tethering-associated proteins can be measured using a proximation ligation assay (PLA). In PLA, two primary antibodies of different species are used to detect unique proteins of interest. Oligonucleotide-labeled secondary antibodies (PLA probes) then bind to the primary antibodies, and PLA probes in close proximity are joined by hybridizing connector oligos, forming a closed-circle DNA template that is then amplified by DNA polymerase. Complementary detection oligos coupled to fluorophores then hybridize to the repeating sequences, which can be imaged using microscopy. Co-immunoprecipitation (Co-IP), ER Tracker, and MitoTracker can also be used to examine changes in MERC colocalization in the absence or presence of genes of interest and treatment conditions ${ }^{4}$. ER Tracker is an ER-specific dye that shares some overlap with mitochondria. Mitochondrial staining may be used to justify the differences in colocalization and changes in TEM analyses. MERC distance can be measured by examining the ER-mitochondria contact space distance or coverage using focused ion beam scanning electron microscopy ${ }^{16}$, electron tomography ${ }^{17}$, or immunogold labeling ${ }^{19}$, in which a protein known to be associated with MERC tethering or mitochondria is stained individually. The colocalization of these immunogold-labeled dots can also be examined. The immunogold labeling of MFN-1, an outer mitochondrial membrane protein involved in mitochondrial fusion, shows that MFN-1 localizes to the mitochondria. These techniques can also be harnessed to validate changes in MERC tethering proteins ${ }^{17}$, similar to PLA $^{20,21}$.

\section{Conclusion}

The present study demonstrates an optimal approach for preserving specimens and measuring organelle morphology using serial-section imaging with TEM. We demonstrated methods that induce hypoxic conditions can introduce unintentional artifacts. We also describe optimal conditions for fixing cells in culture to preserve cellular and mitochondrial integrity. Furthermore, this study presents standardized 
quantifications that can be used to measure mitochondrial morphology and other organellar structures. Finally, we verified previously studied phenotypes to illustrate the efficacy of our methodology. Using this method, researchers should be able to visualize ultrastructure accurately and reproducibly in cells and tissues using TEM imaging techniques.

Online Methods (provides all technical details necessary for the independent reproduction of the methodology, without referring to a chain of bibliographical references). 


\section{References}

1. Cui, L. \& Liu, P. Two Types of Contact Between Lipid Droplets and Mitochondria. Front. Cell Dev. Biol. 8, 618322 (2020).

2. Chandel, N. S. Evolution of Mitochondria as Signaling Organelles. Cell Metabolism 22, 204-206 (2015).

3. Quirós, P. M., Mottis, A. \& Auwerx, J. Mitonuclear communication in homeostasis and stress. Nat Rev Mol Cell Biol 17, 213-226 (2016).

4. Parra, V. et al. Insulin Stimulates Mitochondrial Fusion and Function in Cardiomyocytes via the AktmTOR-NF B-Opa-1 Signaling Pathway. Diabetes 63, 75-88 (2014).

5. Naon, D. et al. Critical reappraisal confirms that Mitofusin 2 is an endoplasmic reticulummitochondria tether. Proc Natl Acad Sci USA 113, 11249-11254 (2016).

6. Favaro, G. et al. DRP1-mediated mitochondrial shape controls calcium homeostasis and muscle mass. Nat Commun 10, 2576 (2019).

7. Frezza, C. et al. OPA1 Controls Apoptotic Cristae Remodeling Independently from Mitochondrial Fusion. Cell 126, 177-189 (2006).

8. Patra, M., Mahata, S. K., Padhan, D. K. \& Sen, M. CCN6 (Wnt induced signaling protein - 3) regulates mitochondrial function. Journal of Cell Science jcs.186247 (2016) doi:10.1242/jcs.186247.

9. Eisner, V. et al. Mitochondrial fusion dynamics is robust in the heart and depends on calcium oscillations and contractile activity. Proc Natl Acad Sci USA 114, E859-E868 (2017).

10. Giacomello, M. \& Pellegrini, L. The coming of age of the mitochondria-ER contact: a matter of thickness. Cell Death Differ 23, 1417-1427 (2016).

11. Csordás, G., Weaver, D. \& Hajnóczky, G. Endoplasmic Reticulum-Mitochondrial Contactology: Structure and Signaling Functions. Trends in Cell Biology 28, 523-540 (2018).

12. Filadi, R. et al. Mitofusin 2 ablation increases endoplasmic reticulum-mitochondria coupling. Proc Natl Acad Sci USA 112, E2174-E2181 (2015). 
13. Kowaltowski, A. J. et al. Mitochondrial morphology regulates organellar $\mathrm{Ca}^{2+}$ uptake and changes cellular $\mathrm{Ca}^{2+}$ homeostasis. The FASEB Journal 33, 13176-13188 (2019).

14. Hirabayashi, Y. et al. ER-mitochondria tethering by PDZD8 regulates $\mathrm{Ca}^{2+}$ dynamics in mammalian neurons. Science 358, 623-630 (2017).

15. Sood, A. et al. A Mitofusin-2-dependent inactivating cleavage of Opa1 links changes in mitochondria cristae and ER contacts in the postprandial liver. Proc Natl Acad Sci USA 111, 16017$16022(2014)$.

16. Glancy, B. et al. Mitochondrial reticulum for cellular energy distribution in muscle. Nature 523, 617$620(2015)$.

17. Tsushima, K. et al. Mitochondrial Reactive Oxygen Species in Lipotoxic Hearts Induce PostTranslational Modifications of AKAP121, DRP1, and OPA1 That Promote Mitochondrial Fission. Circ Res 122, 58-73 (2018).

18. Muñoz, J. P. et al. Mfn2 modulates the UPR and mitochondrial function via repression of PERK. EMBO J 32, 2348-2361 (2013).

19. Galmes, R. et al. ORP5/ORP8 localize to endoplasmic reticulum-mitochondria contacts and are involved in mitochondrial function. EMBO Rep 17, 800-810 (2016).

20. Tubbs, E. et al. Disruption of Mitochondria-Associated Endoplasmic Reticulum Membrane (MAM) Integrity Contributes to Muscle Insulin Resistance in Mice and Humans. Diabetes 67, 636-650 (2018).

21. Thoudam, T. et al. PDK4 Augments ER-Mitochondria Contact to Dampen Skeletal Muscle Insulin Signaling During Obesity. Diabetes 68, 571-586 (2019).

22. Zhang, L. et al. Altered brain energetics induces mitochondrial fission arrest in Alzheimer's Disease. Sci Rep 6, 18725 (2016).

23. Palade, G. E. AN ELECTRON MICROSCOPE STUDY OF THE MITOCHONDRIAL STRUCTURE. J Histochem Cytochem. 1, 188-211 (1953). 
24. Sjöstrand, F. S. Electron Microscopy of Mitochondria and Cytoplasmic Double Membranes: UltraStructure of Rod-shaped Mitochondria. Nature 171, 30-31 (1953).

25. Bullock, G. R. The current status of fixation for electron microscopy: A review. Journal of Microscopy 133, 1-15 (1984).

26. White, D. L., Mazurkiewicz, J. E. \& Barrnett, R. J. A chemical mechanism for tissue staining by osmium tetroxide-ferrocyanide mixtures. J Histochem Cytochem. 27, 1084-1091 (1979).

27. Principles and techniques of electron microscopy: biological applications. (Cambridge University Press, 2000).

28. Wisse, E. Fixation methods for electron microscopy of human and other liver. WJG 16, 2851 (2010).

29. Boyde, A. \& Wood, C. Preparation of animal tissues for surface-scanning electron microscopy. Journal of Microscopy 90, 221-249 (1969).

30. Braet, F., De Zanger, R. \& Wisse, E. Drying cells for SEM, AFM and TEM by hexamethyldisilazane: a study on hepatic endothelial cells. Journal of Microscopy 186, 84-87 (1997).

31. Nation, J. L. A New Method Using Hexamethyldisilazane for Preparation of Soft Insect Tissues for Scanning Electron Microscopy. Stain Technology 58, 347-351 (1983).

32. Arborgh, B., Bell, P., Brunk, U. \& Collins, V. P. The osmotic effect of glutaraldehyde during fixation. A transmission electron microscopy, scanning electron microscopy and cytochemical study. Journal of Ultrastructure Research 56, 339-350 (1976).

33. Desmet, V. J. The amazing universe of hepatic microstructure. Hepatology 50, 333-344 (2009).

34. Schwerzmann, K., Hoppeler, H., Kayar, S. R. \& Weibel, E. R. Oxidative capacity of muscle and mitochondria: correlation of physiological, biochemical, and morphometric characteristics. Proceedings of the National Academy of Sciences 86, 1583-1587 (1989).

35. Whelan, D. R. \& Bell, T. D. M. Image artifacts in Single Molecule Localization Microscopy: why optimization of sample preparation protocols matters. Sci Rep 5, 7924 (2015).

36. Montero Llopis, P. et al. Best practices and tools for reporting reproducible fluorescence microscopy methods. Nat Methods (2021) doi:10.1038/s41592-021-01156-w. 
37. Hekmatshoar, Y., Nakhle, J., Galloni, M. \& Vignais, M.-L. The role of metabolism and tunneling nanotube-mediated intercellular mitochondria exchange in cancer drug resistance. Biochemical Journal 475, 2305-2328 (2018).

38. Vignais, M.-L., Caicedo, A., Brondello, J.-M. \& Jorgensen, C. Cell Connections by Tunneling Nanotubes: Effects of Mitochondrial Trafficking on Target Cell Metabolism, Homeostasis, and Response to Therapy. Stem Cells International 2017, 1-14 (2017).

39. Wang, X. \& Gerdes, H.-H. Transfer of mitochondria via tunneling nanotubes rescues apoptotic PC12 cells. Cell Death Differ 22, 1181-1191 (2015).

40. Vincent, A. E., Turnbull, D. M., Eisner, V., Hajnóczky, G. \& Picard, M. Mitochondrial Nanotunnels. Trends in Cell Biology 27, 787-799 (2017).

41. Zhou, Z. et al. Endoplasmic reticulum-associated degradation regulates mitochondrial dynamics in brown adipocytes. Science 368, 54-60 (2020).

42. Fecher, C. et al. Cell-type-specific profiling of brain mitochondria reveals functional and molecular diversity. Nat Neurosci 22, 1731-1742 (2019). 
$\mathrm{CO} 2$


$5 \%$ isoflurane $/ \mathrm{O}_{2}$

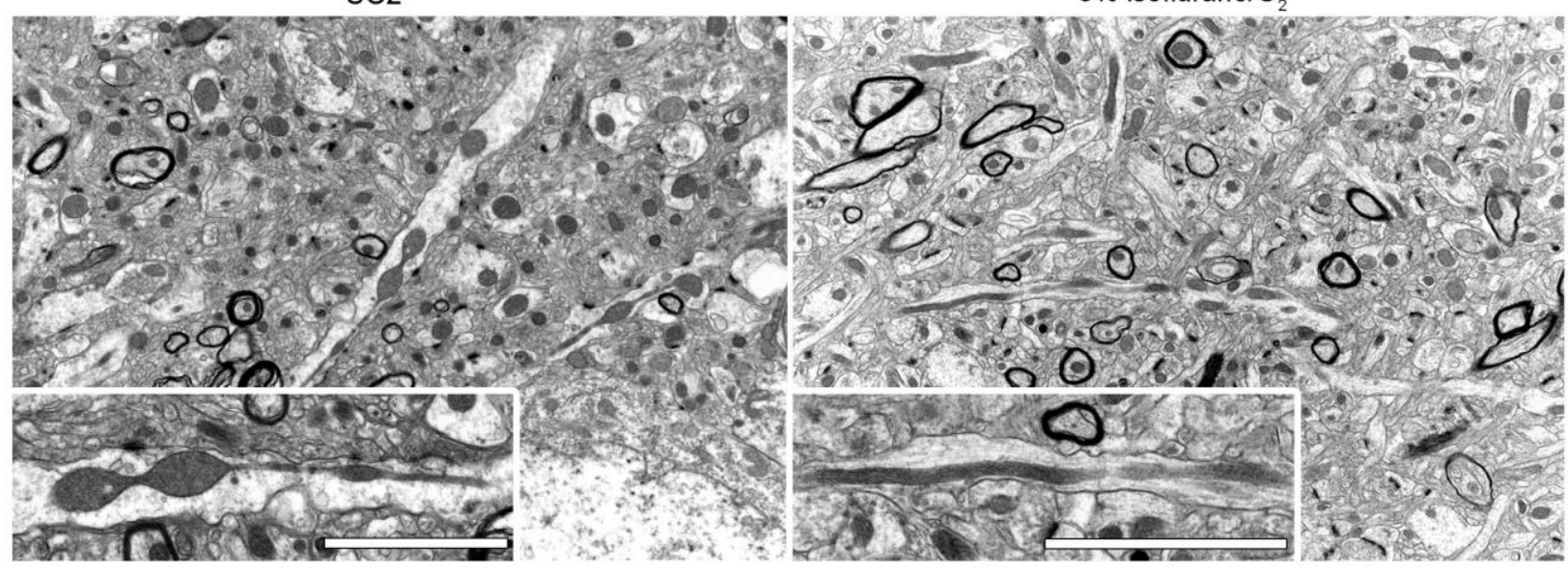

$\underline{\text { anesthesia }}$

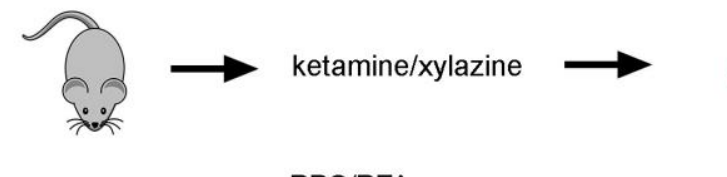

perfusion



PFA only

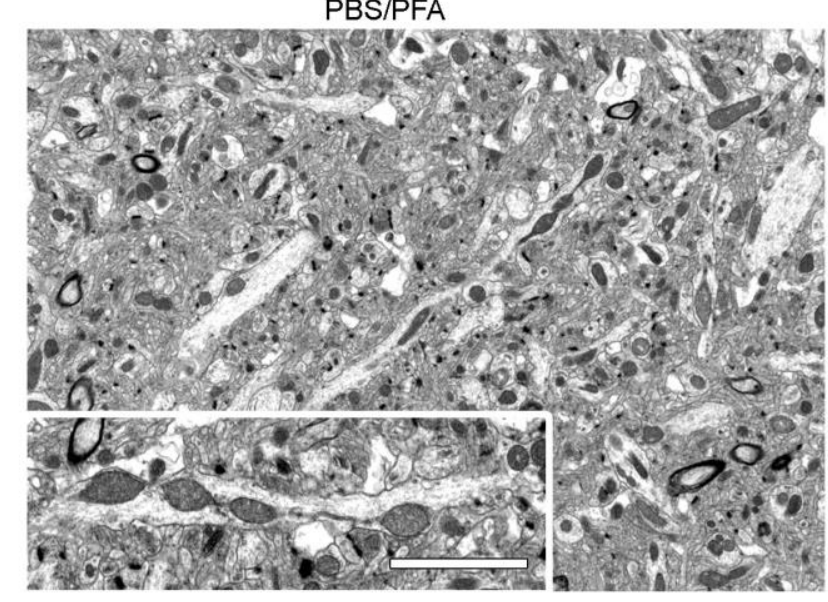

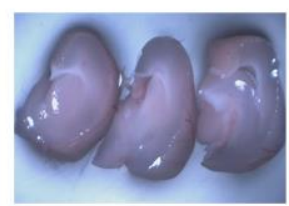

immersion fix $1 \mathrm{~mm}$ slices of brain



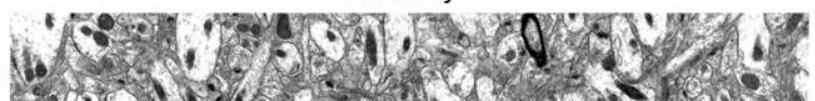

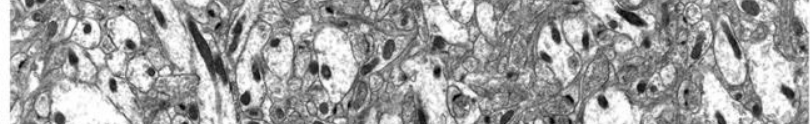

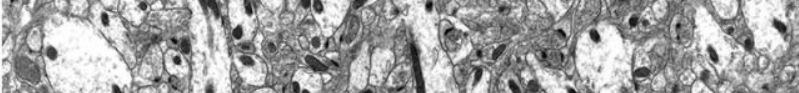

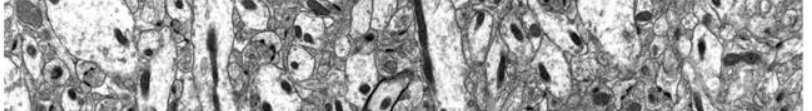



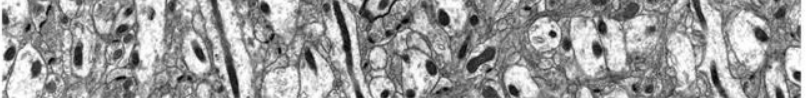

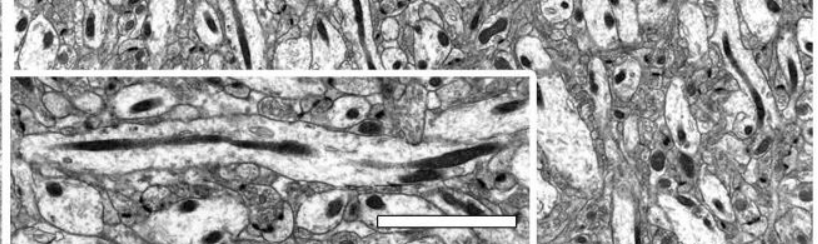
1) 30 En $: N$

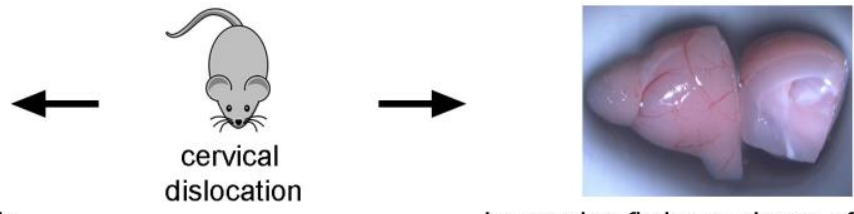

immersion fix large pieces of brain

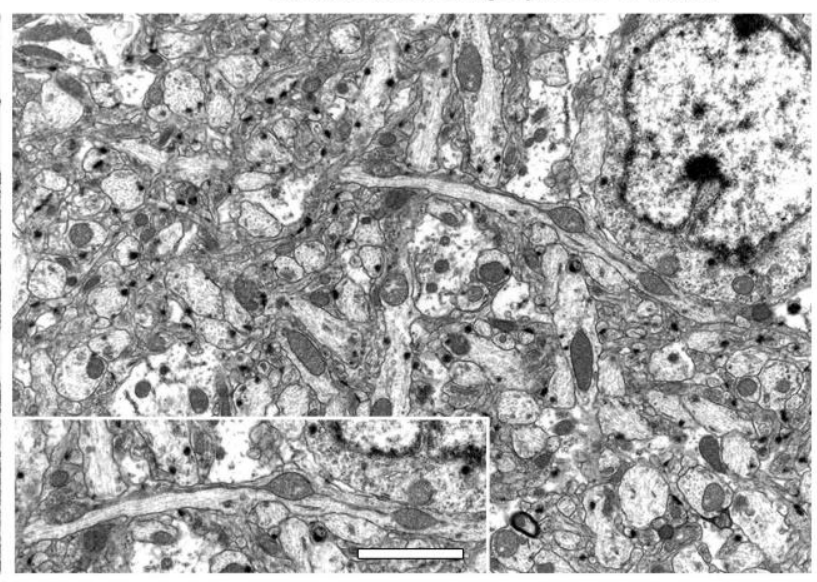




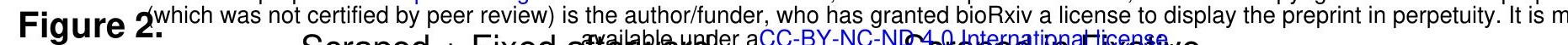

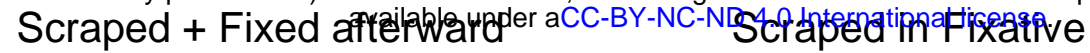


5 min Fixative + Scraping

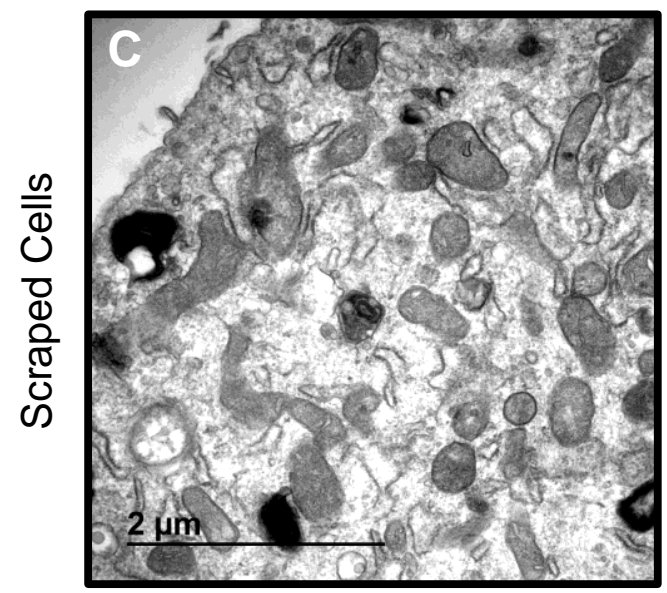

10 min Fixative + Scraping
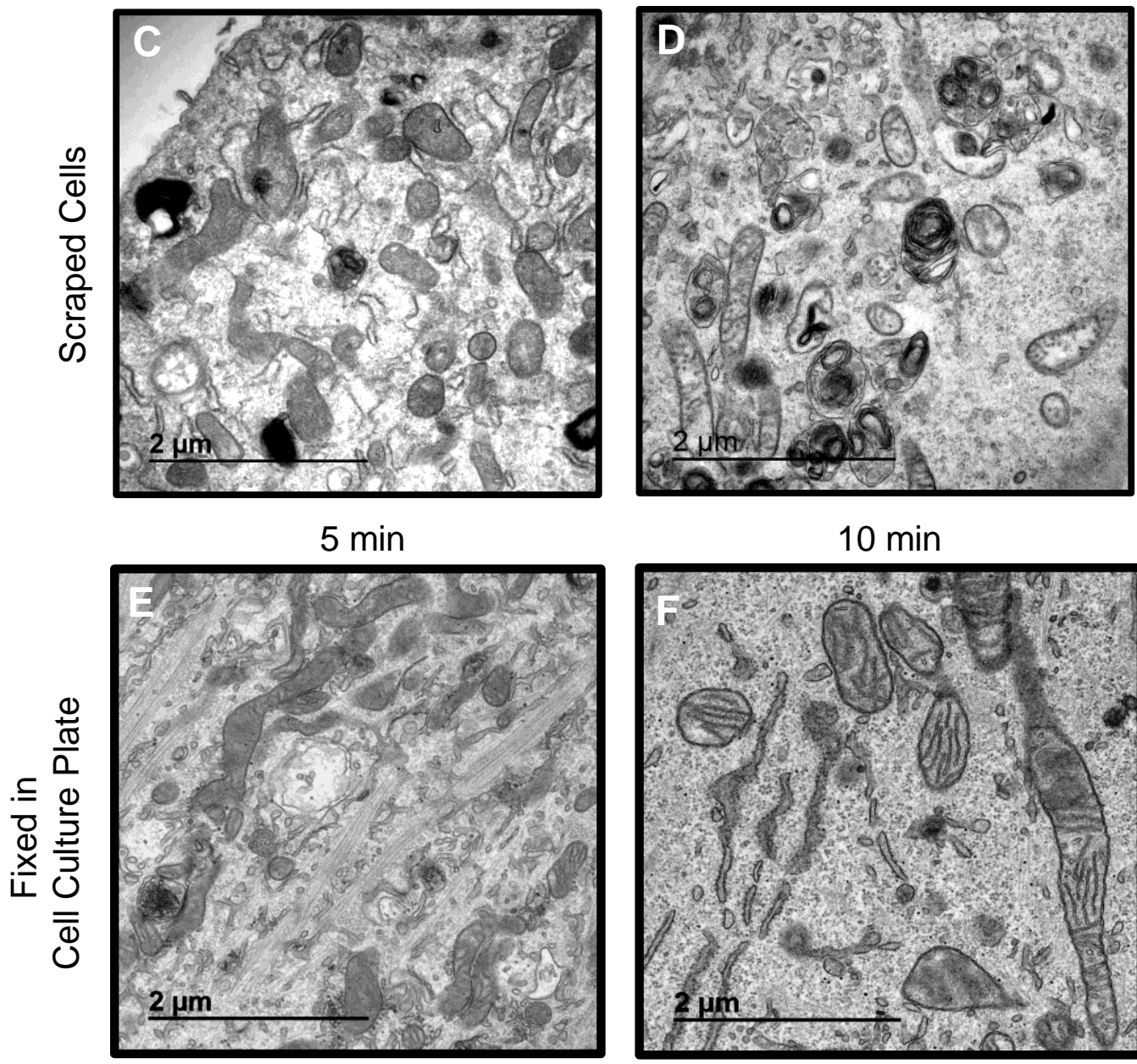

$10 \min$
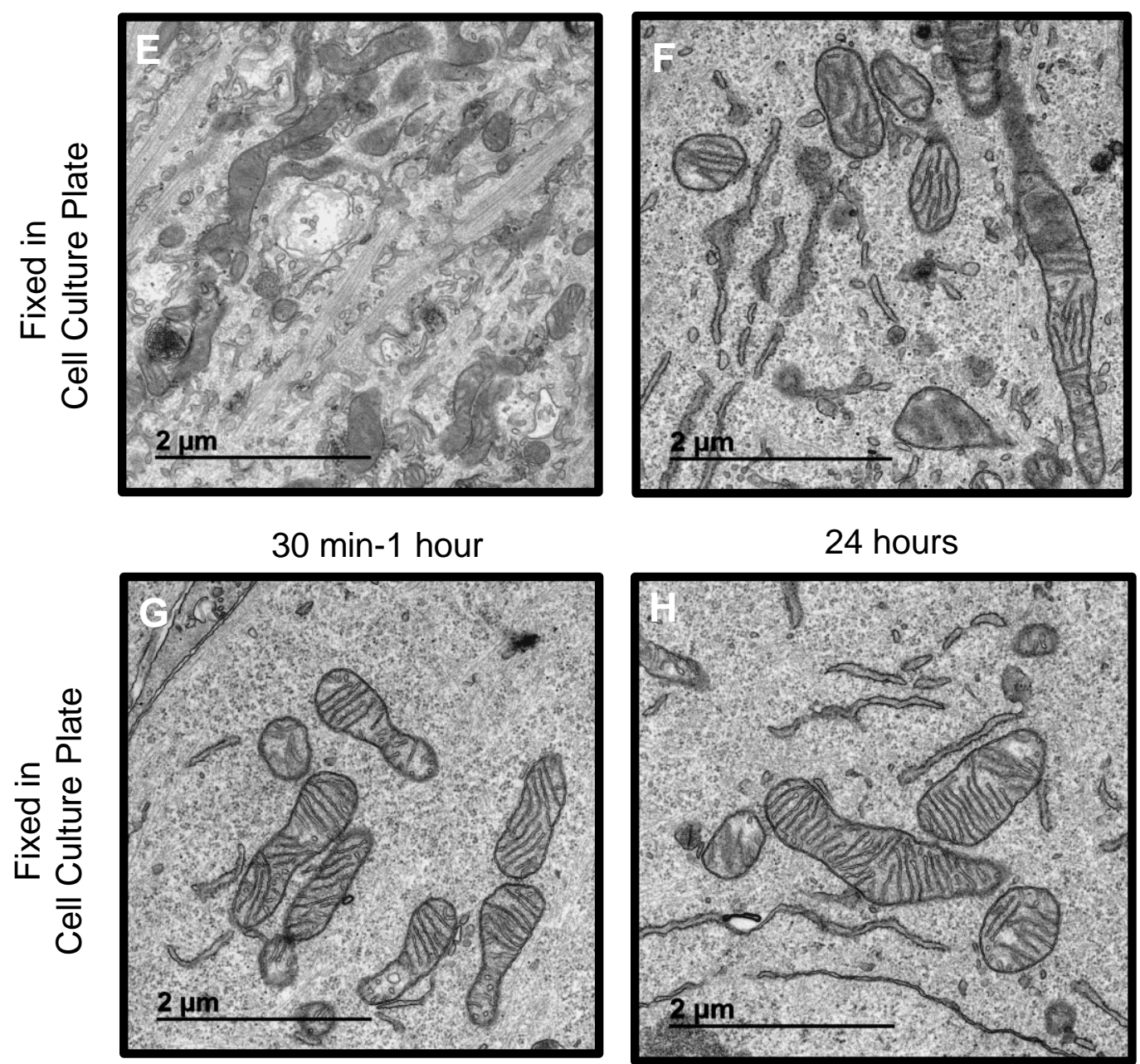

24 hours

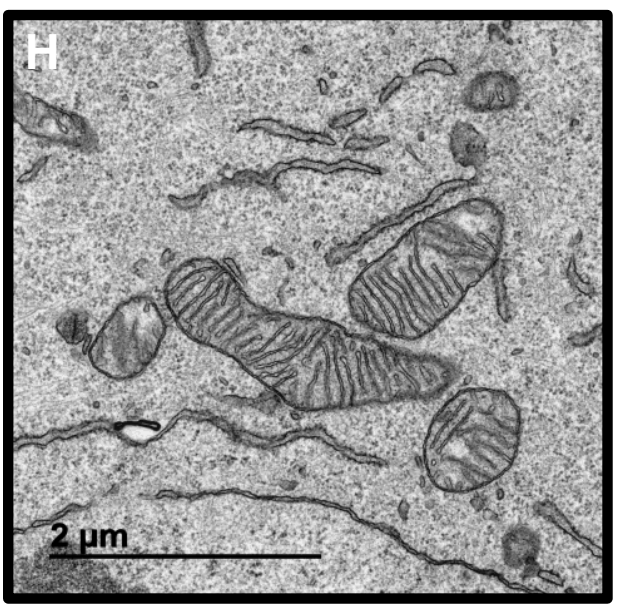


Figure 3.
which was not certified by peer review) is the author/funder, who has granted bioRxiv a license to display the preprint in perpetuity. It is $m$
available under aCC-BY-NC-ND 4.0 International license.


B

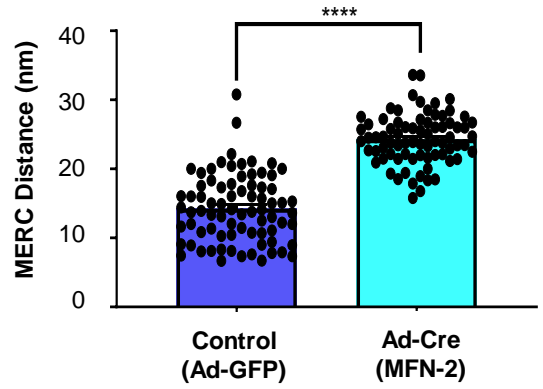

C
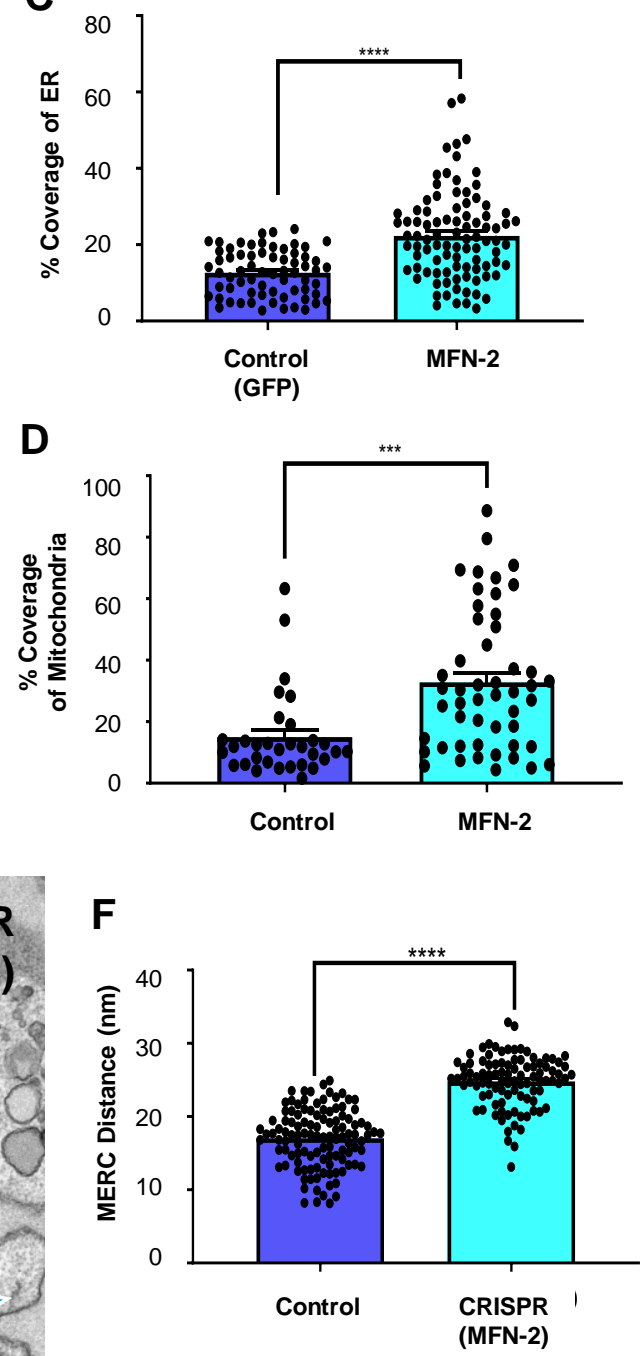

H

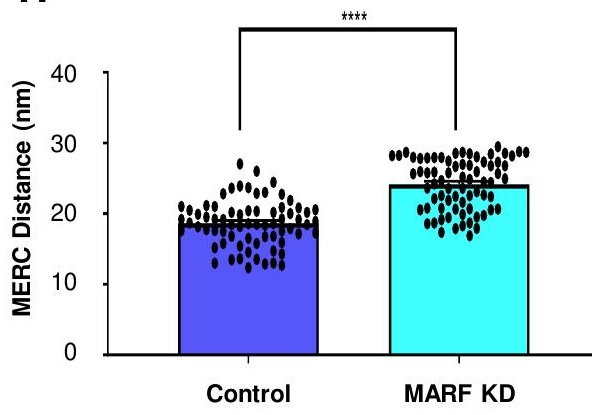


Figure 4.

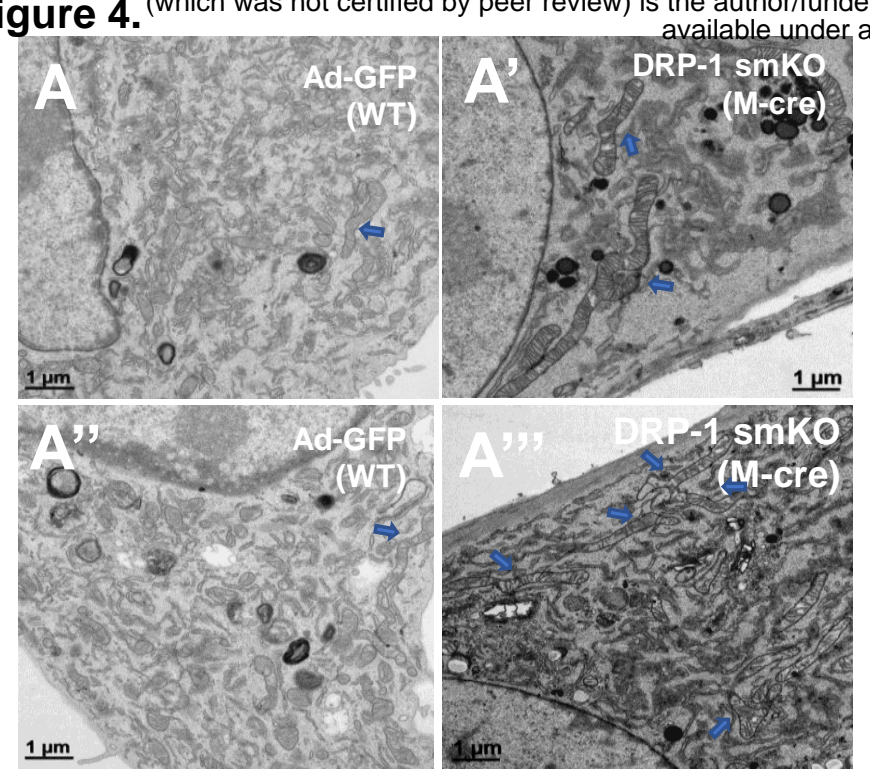

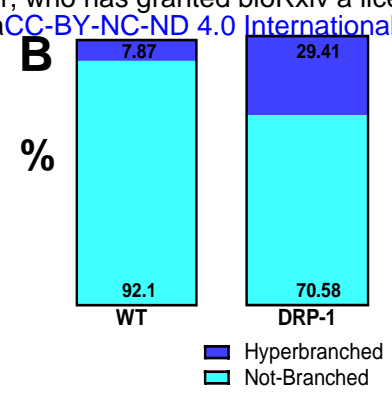
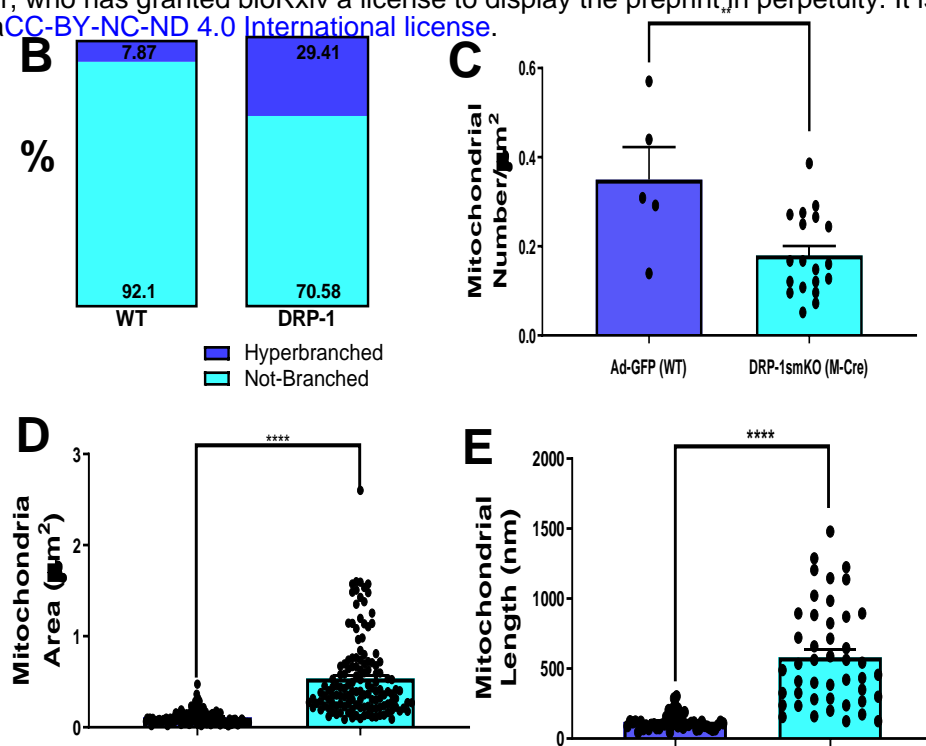

Ad-GFP (WT) DRP-1smKO (M-Cre)

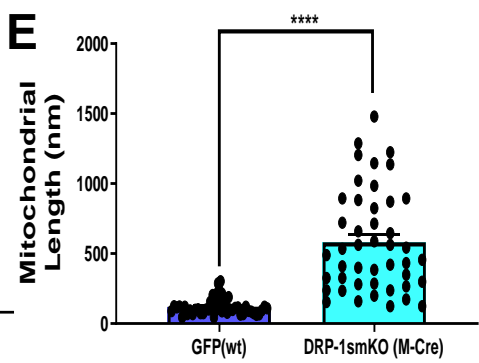

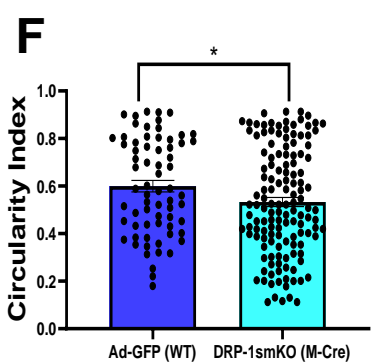


Ad-GFP

Ad-GFP 


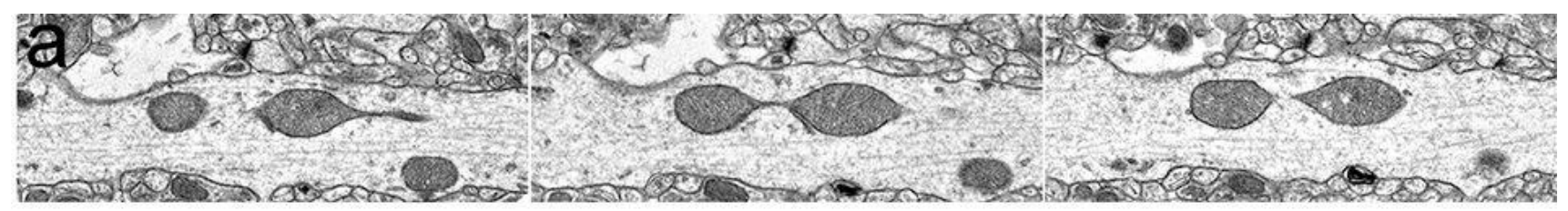

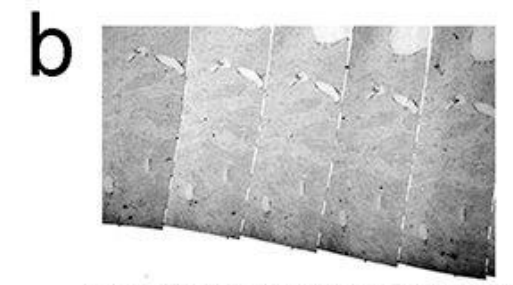

serial sections on formvar coated slot grids

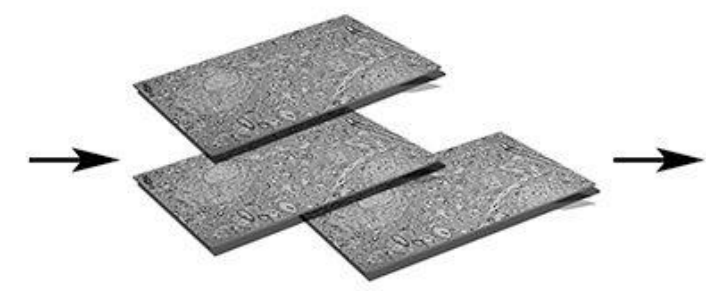

collect image stack

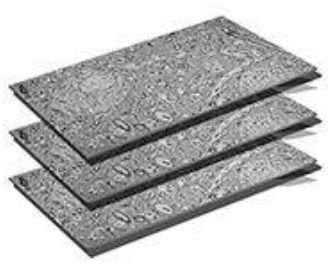

align image stack

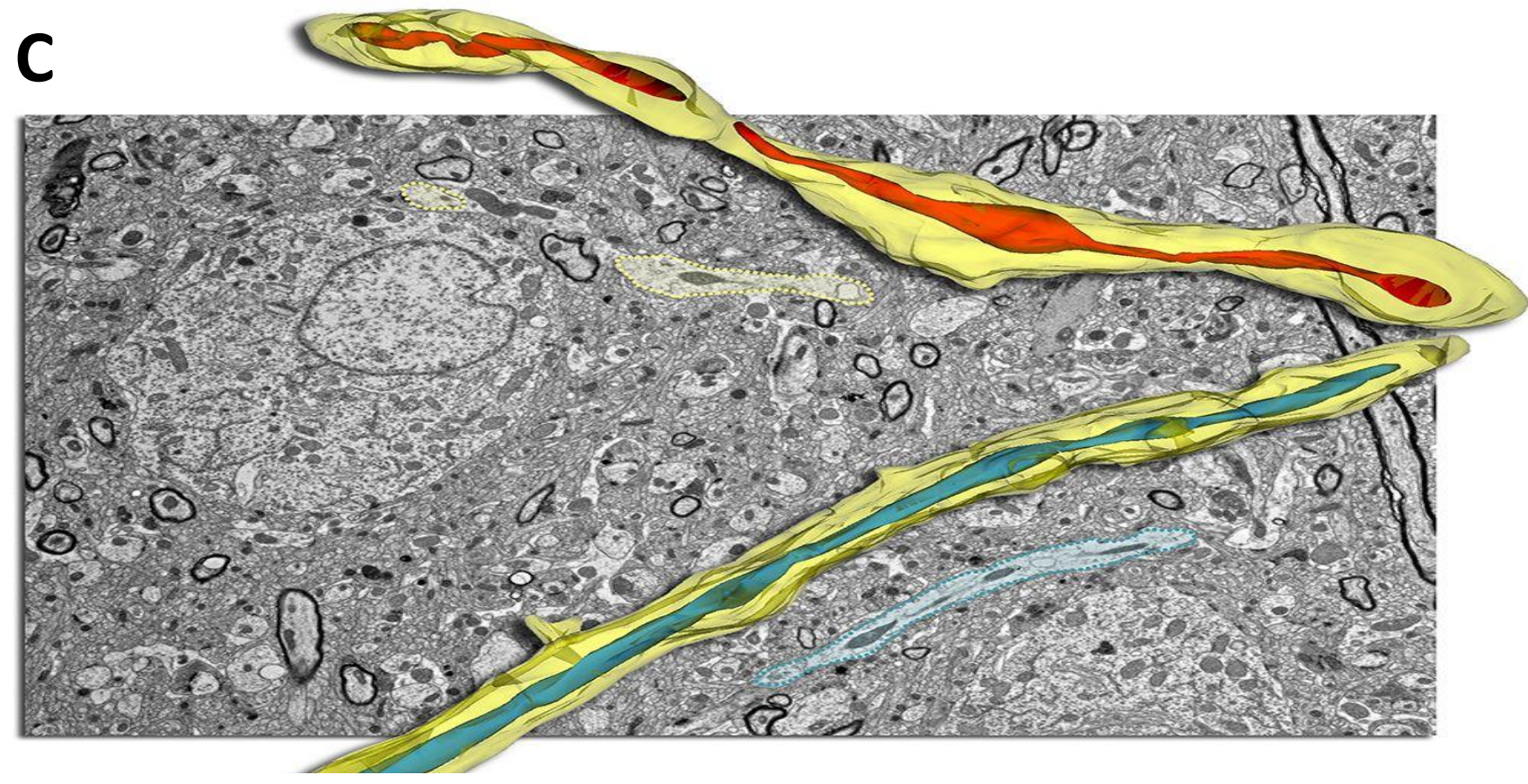

D

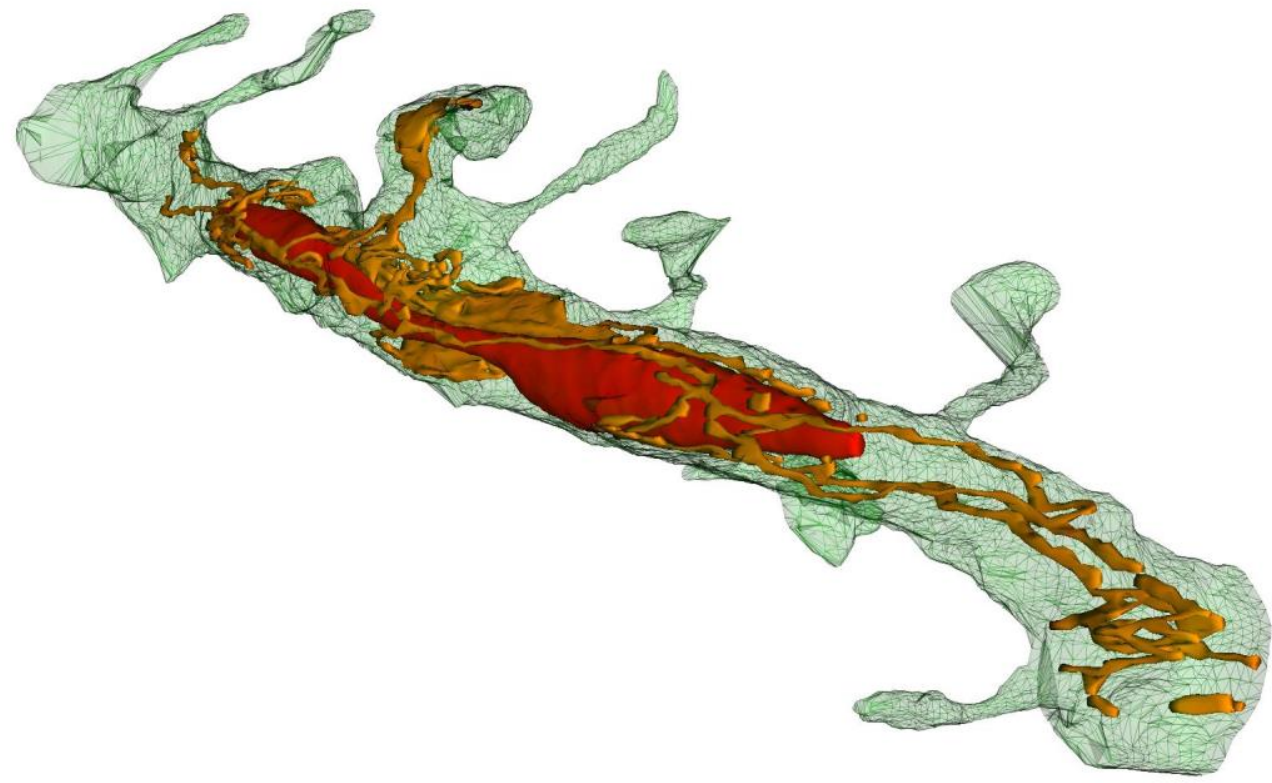


(which was not certified by peer review) is the author/funder, who has granted bioRxiv a license to display the preprint in perpetuity. It is $m$

A Download Image J from NiH website: https://imagej.nih.gov/ij/download.htmı

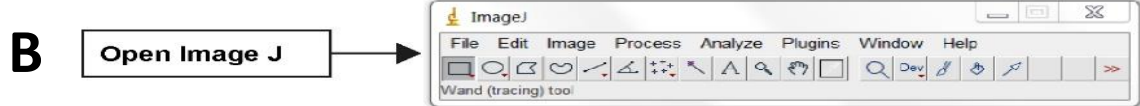

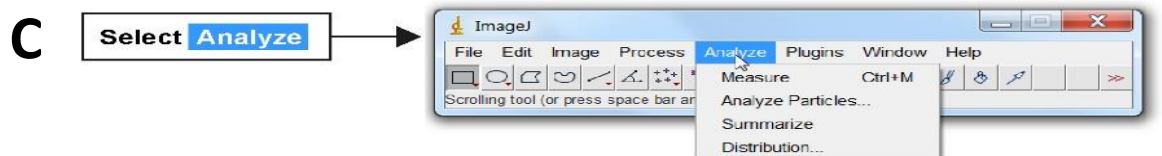
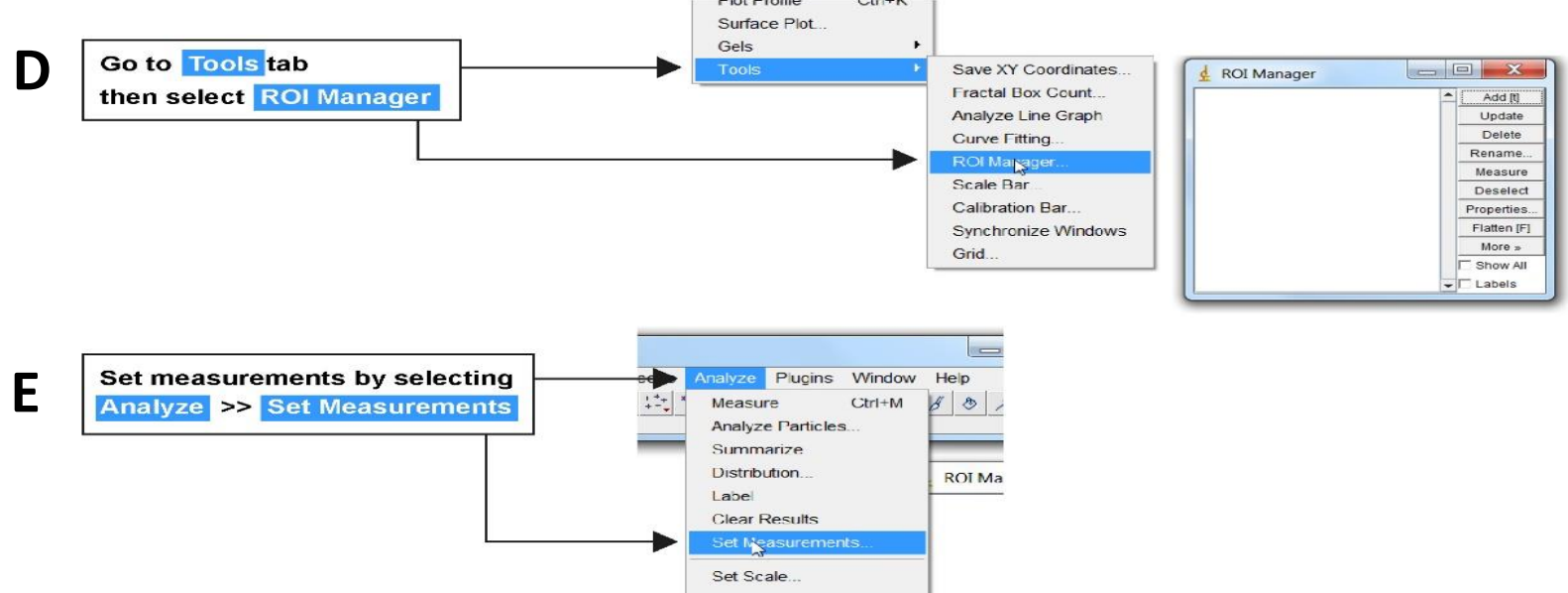

F $\begin{aligned} & \text { Set Measurements box will appear } \\ & \text { Click on all similar images and press "OK" }\end{aligned}$
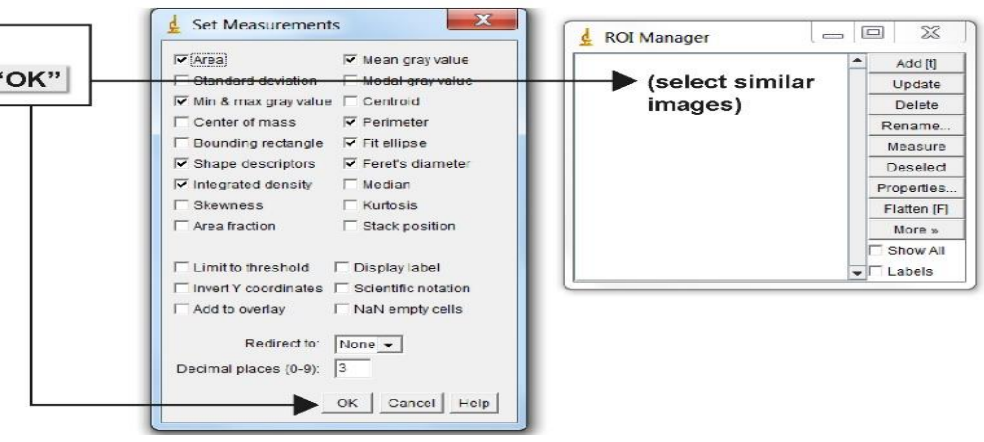

$\mathbf{G}$ Open an image for measuring:

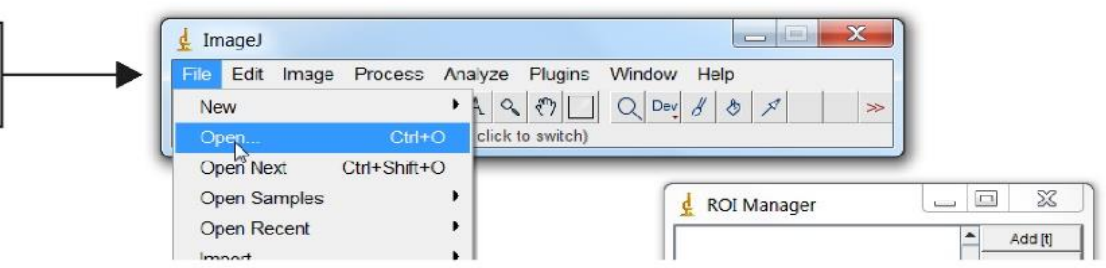
H Select a TIFF or DM3 image to be
uploaded for measurements

Once image appears, measuring of organelle morphology can begin 


\section{Figure S3.}
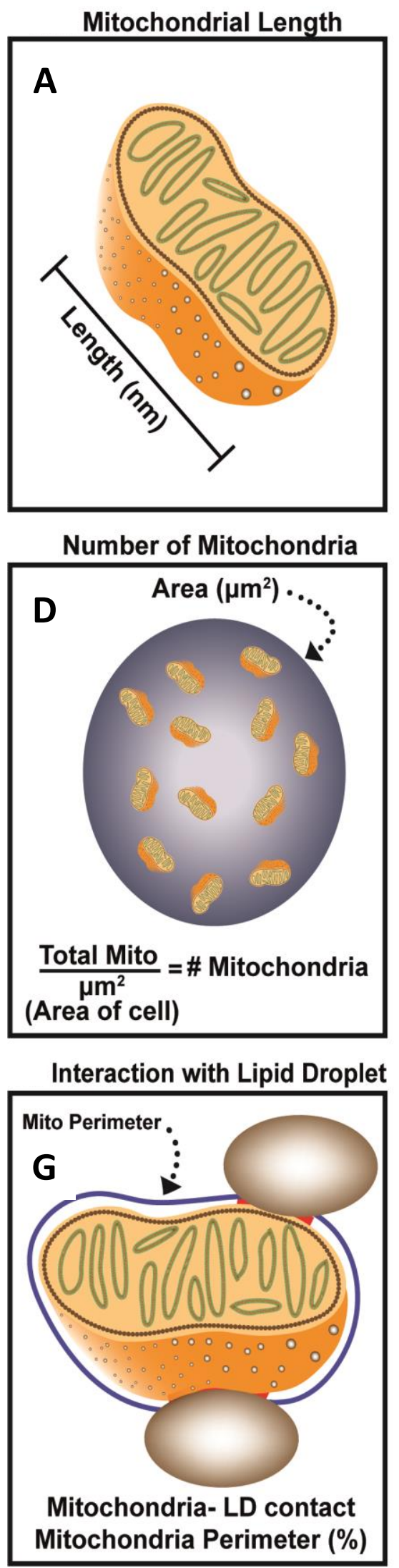
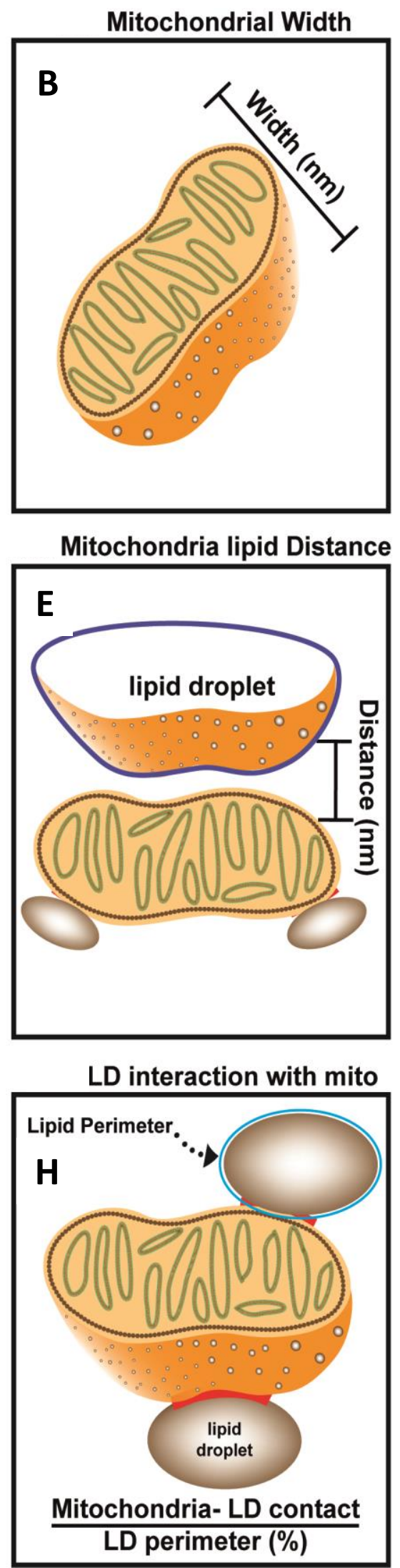

Area, Circularity Index, Perimeter



Mitochondria Volume Density



Mitochondria Lipid Interface

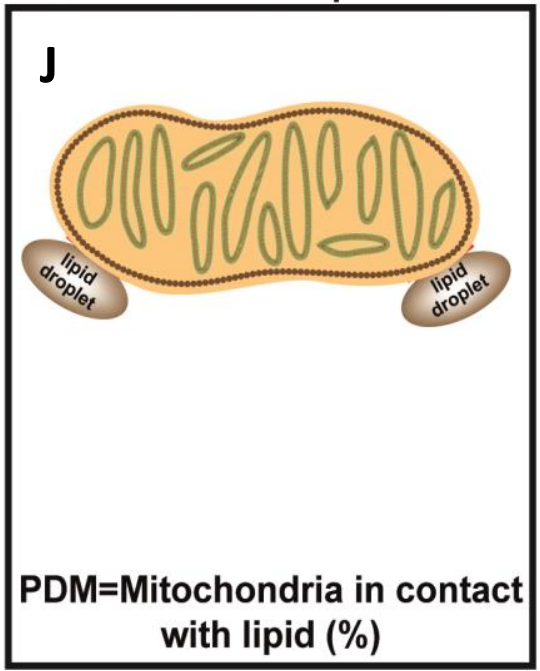




\section{Figure S4.}

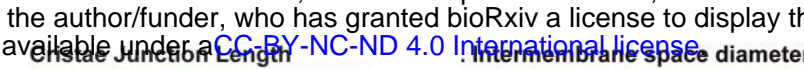
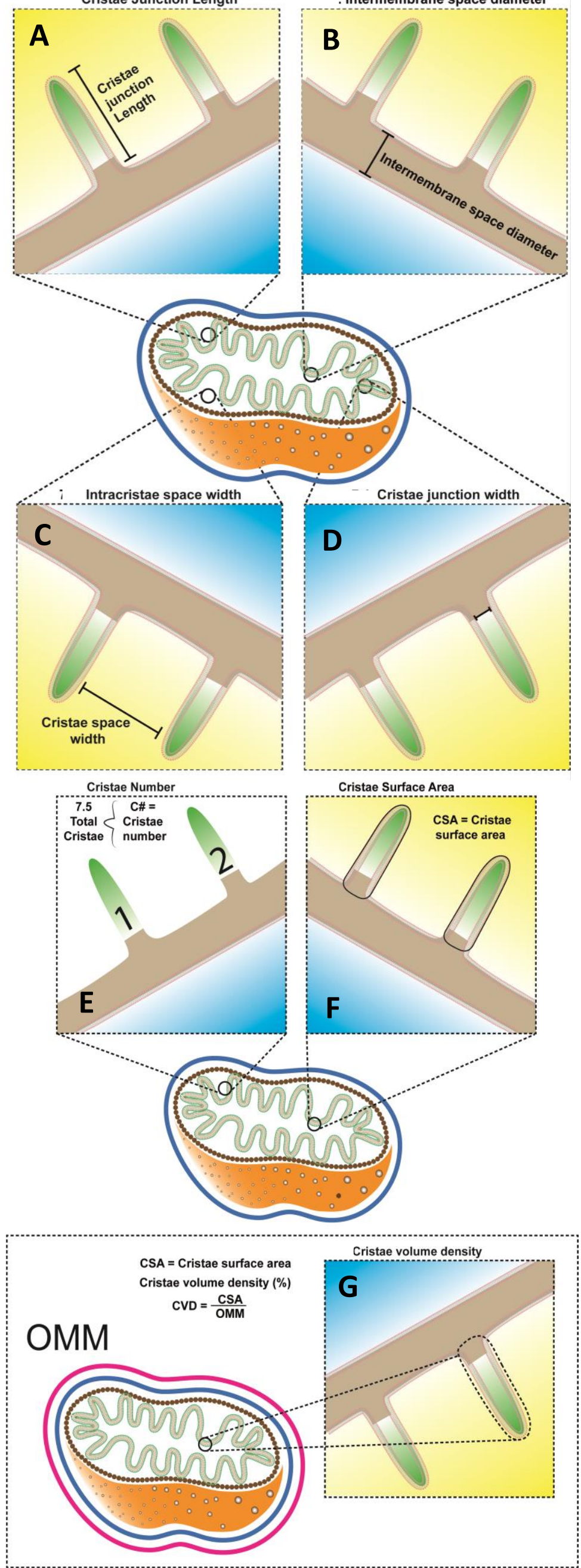
Figure S5.

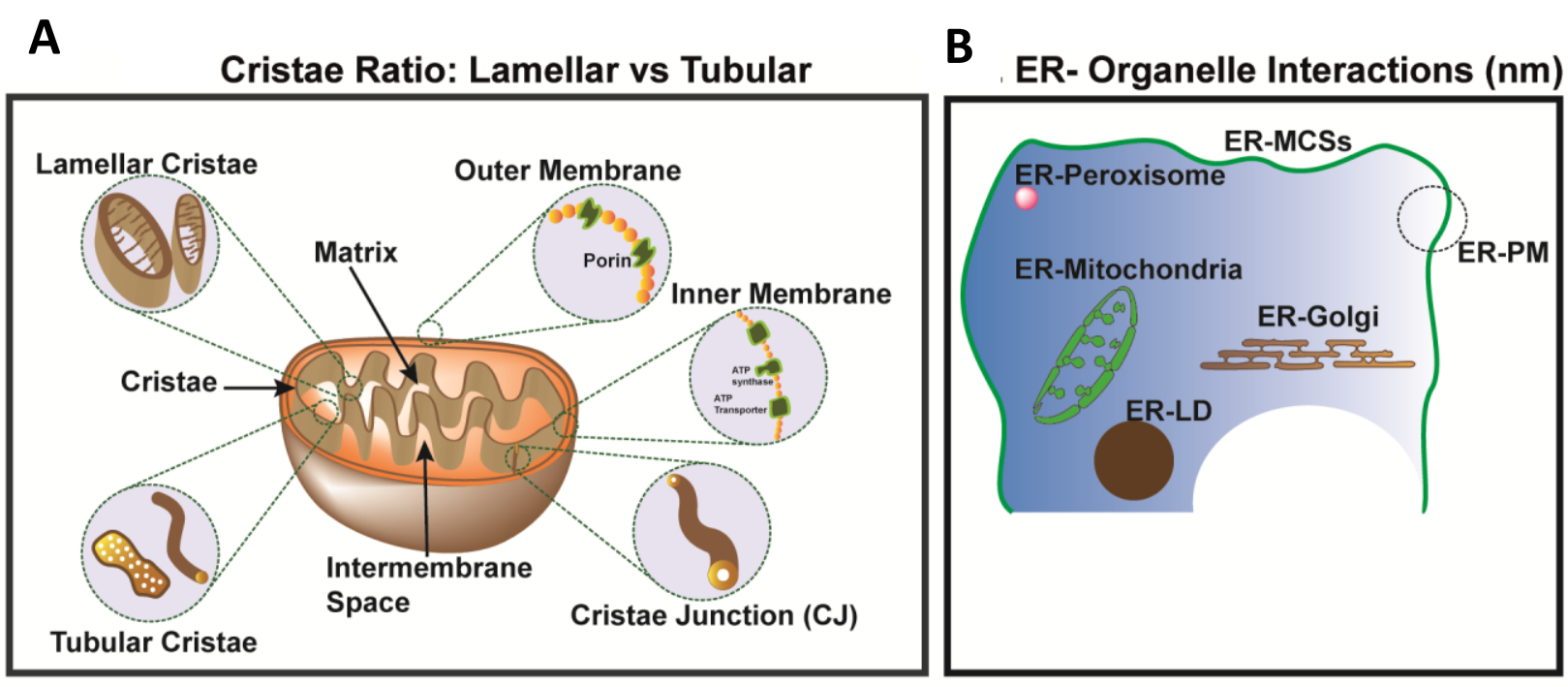

Percent Coverage of MERC

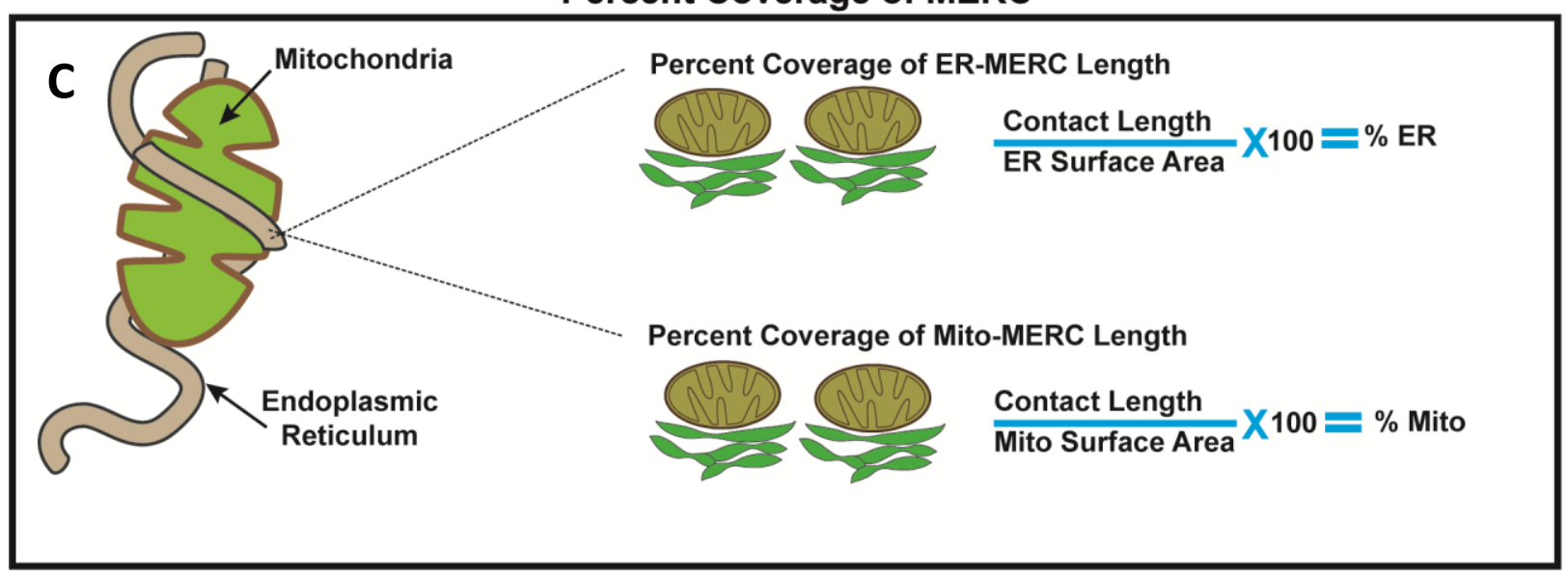


(which was not certified by peer review) is the author/funder, who has granted bioRxiv a license to display the preprint in perpetuity. It is $m$ Figure S6. available under aCC-BY-NC-ND 4.0 International license.
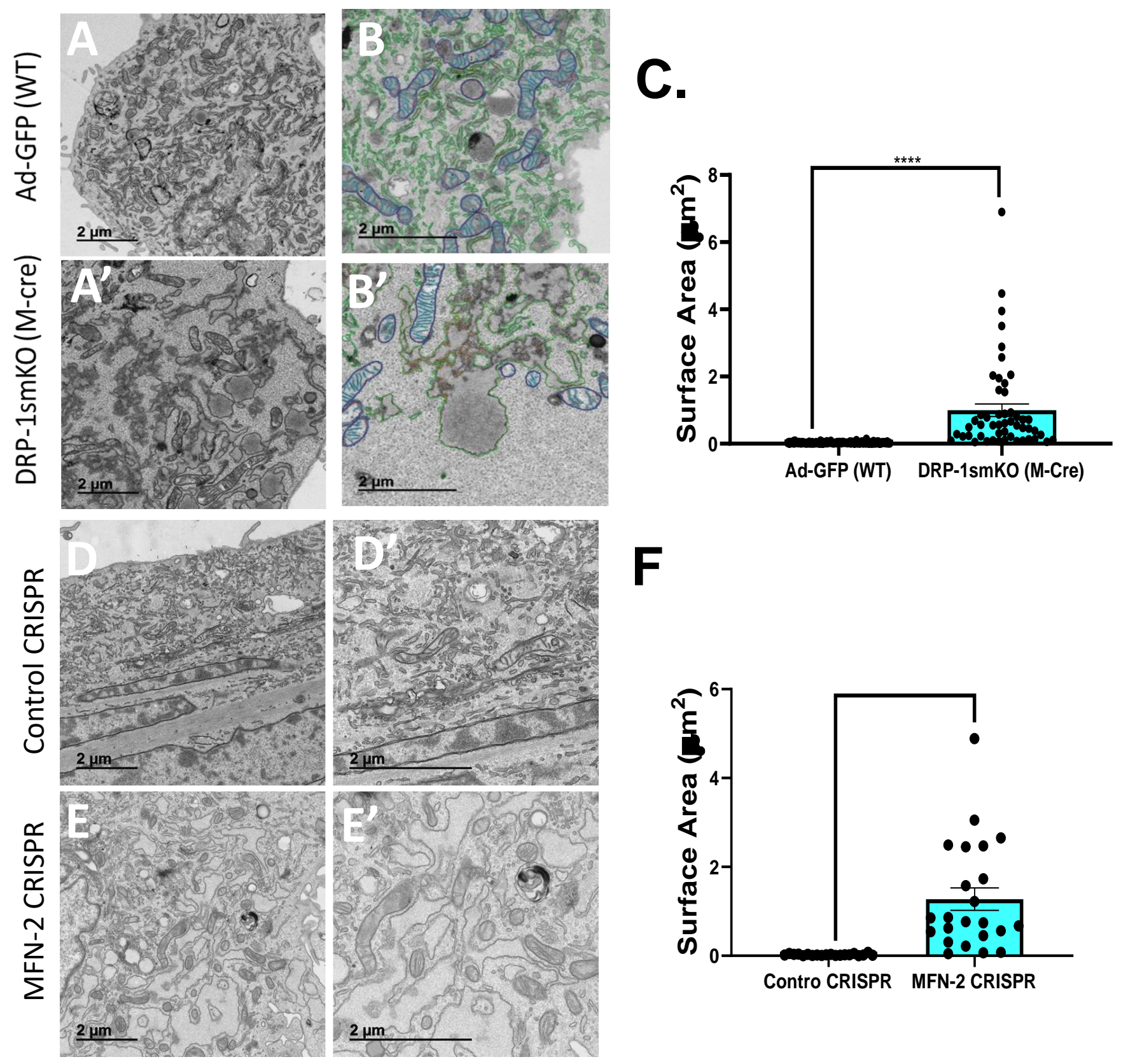

$\mathbf{F}$




Figure S7.

Figure S8.
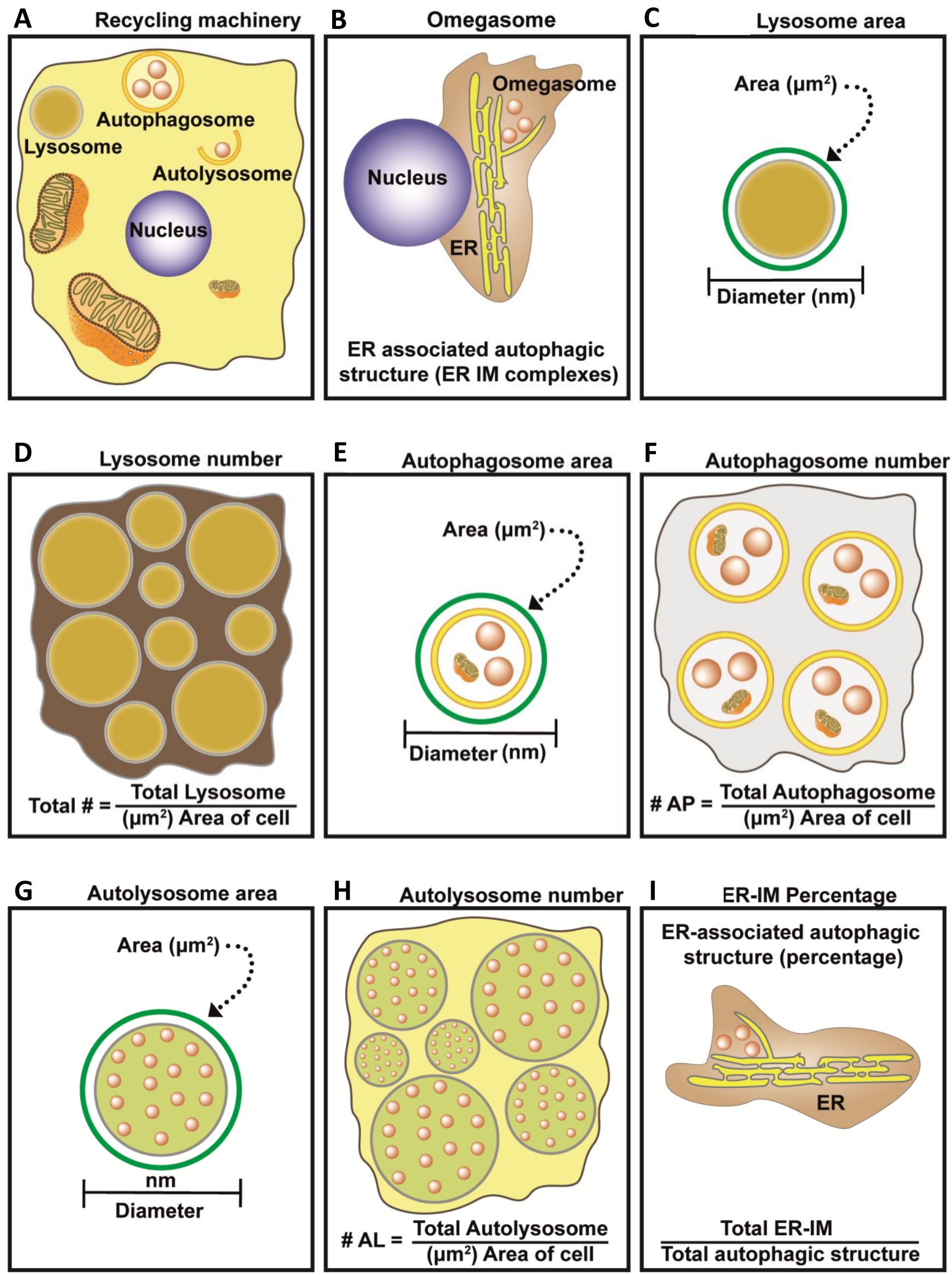
Figure 1. Evaluation of EM processing conditions to prepare artifact-free tissue for assaying mitochondrial morphology. A. Wild type (WT) mice were sacrificed by cervical dislocation after anesthesia with $\mathrm{CO}_{2}$ or $5 \%$ isoflurane/oxygen inhalation. Fresh brains were removed and cut into coronal sections; the CA1 hippocampal region (hipp) from each section was dissected and further processed for the TEM or SEM. B. $\mathrm{CO}_{2}$ exposure for 3 min was sufficient to induce MOAS formation in WT hipp tissue. C. Hipp mitochondria, from mice administered 5\% isoflurane before cervical dislocation and subsequent dissection, maintain normal shape and size that were typical for WT mice. D. WT mice were anesthetized using ketamine/xylazine followed by cardiac perfusion with a PBS and 4\% PFA flush, or with 4\% PFA only. Mice were sacrificed by cervical dislocation; brains were removed and fixed in Trump's solution overnight. The next day, the brain was cut into coronal sections; CA1 hipp region from each section was dissected as shown in (a) and subjected to further processing for the TEM or SEM. E, F. Cardiac perfusion preceded with the PBS flush induced MOAS formation in WT hipp tissue. The perfusion without PBS flush did not cause MOAS formation. G. WT mice were sacrificed by cervical dislocation without prior anesthesia. The brain was removed; one hemisphere was cut in $1 \mathrm{~mm}$-thick slices (thin, left), while the other hemisphere was cut in two halves (thick, right). Tissues were subjected to immersion fixation in Trump's solution overnight. Tissues cut into thin slices were free of MOAS $(\mathbf{G , H})$, while larger pieces of tissue displayed pronounced MOAS formation (I). Scale bars, $2 \mu \mathrm{m}$.

Figure 2. Comparison of cultured cell harvesting and fixation methods based on mitochondrial morphology. Representative micrographs of cultured cells A. scraped followed by fixation, B. scraped in TEM fixative; C. scraping after 5 minutes in TEM fixative; D. scraping after 10 minutes in TEM fixative; E. 5 minutes in TEM fixative without scraping; F. 10 minutes in TEM fixative without scraping; G. 30 to 60 mins in TEM fixative without scraping; H. 24 hours in TEM fixative without scraping. All cultured cells were myotubes treated with insulin for 2 hours before fixation.

Figure 3. MERC ultrastructure in MFN-2-deficient primary fibroblasts and MARF-deficient Drosophila cells. A-A'. Control and MFN-2 deficient primary fibroblasts were imaged using TEM at 
15,000x and 20,000x magnification, then MERC features were quantified. B. MERC distance in control and MFN-2 deficient primary fibroblasts. C. \% ER coverage in control and MFN-2 deficient primary fibroblasts. D. \% mitochondria coverage in control and MFN-2 deficient primary fibroblasts. Ultrastructure of E. control CRISPR and E'. CRISPR generated MFN-2 deficient primary myotubes. F. MERC distance in control CRISPR and MFN-2 deficient primary myotubes. Ultrastructure of G. control mito-GFP and G.' MARF-deficient Drosophila. H. MERC distance in control mito-GFP and MARFdeficient Drosophila. Scale bars: A, A', $500 \mu \mathrm{m} ; \mathbf{E}, \mathbf{E}, 2 \mu \mathrm{m} ; \mathbf{G}, \mathbf{G}^{\prime}, 0.2 \mu \mathrm{m}$.

Figure 4. Quantification of mitochondrial features in DRP-1 deficient, insulin-treated, and OPA-1 deficient skeletal muscle myoblasts. Ultrastructure of hyperbranching in A, A', control and A'-A',' DRP-1 deficient primary skeletal myoblasts. Hyperbranching (blue arrows). B. \% hyperbranching, C. mitochondrial number, D. mitochondrial area, E. mitochondrial length, F. circularity index in control and DRP-1 deficient primary skeletal myoblasts. Ultrastructure of G. untreated and G'. 2-hr-insulin-treated primary human myoblasts. H. Mitochondria area, I. cristae score, J. cristae number, K. cristae volume, L. cristae surface area of untreated and 2-hr-insulin-treated primary human myoblasts. Ultrastructure of $\mathbf{M}$. control and M'. OPA-1 deficient primary skeletal muscle myoblasts. N. Mitochondria area, O. cristae score, Q. cristae number, R. cristae volume, S. cristae surface area in control and OPA-1 deficient primary skeletal muscle myoblasts. Scale bars: A-A',', $1 \mu \mathrm{m} ; \mathbf{G}, \mathbf{G}^{\prime}, \mathbf{M}, \mathbf{M}^{\prime}, 2 \mu \mathrm{m}$.

\section{Supplementary Figures}

Figure S1. A. Consecutive serial sections of hippocampi from a wild type (WT) mouse showing mitochondrial fission. B. The major steps that allow 3D TEM reconstruction include serial sectioning, collecting image stack, and aligning the stack to be reconstructed. C. 3D EM reconstruction of mitochondria in the neuropils in the brain of a WT mouse generated using TEM images from $\sim 30$ serial sections $0.09 \square \mu \mathrm{m}$ thick using Reconstruct software. D. TEM images from $\sim 28$ serial sections $0.09 \square \mu \mathrm{m}$ thick were stacked, aligned, and reconstructed using Reconstruct software to depict mitochondria (red) interacting with ER (gold) in a neuropil in the brain of a WT mouse. Scale bar, $2 \mu \mathrm{m}$. 
Figure S2. A flowchart describing the basics of how to use ImageJ software to collect TEM data.

A. Download ImageJ from the NIH website. https.//imagej.nih.gov/ij/download.html. B. Open ImageJ. C. Select "Analyze". D. Go to the "Tools" tab, then select "ROI Manager". E. Set measurements by selecting "Analyze" >> "Set Measurements". F. The "Set Measurements" box will appear. Select the measurements of interest, then click on "all similar images" then click "OK". G. Open an image for measuring. "File" >> "Open". H. Select a TIFF or DM3 image to be uploaded for measurements. I. Measurement of organelle morphology can begine once the image appears.

Figure S3. Model of dimensions associated with mitochondria and mitochondria-lipid interactions.

A. A cartoon depicting the mitochondrial length and major axis. B. Measurement of mitochondrial width and minor axis. C. Representation of mitochondrial area, circularity index, and perimeter. Area is the space occupied by a mitochondrion. Perimeter is the outer boundary of a mitochondrion. The circularity index is the ratio between the areas of an inscribed circle to the area of a circumscribed circle fitted to the outline of a funnel-shaped depression. D. Mitochondrial number using the average of total mitochondrial area and area of the entire cell. E. Mitochondria lipid distance is the space between the outer mitochondrial membrane and the lipid droplet. F Cartoon depicting the mitochondrial volume density, measured by dividing the average total outer mitochondrial membrane area by the area of the entire cell. G. Lipid distance interaction with mitochondria is calculated by subtracting the mitochondria by the lipid distance contact, then dividing by the percentage of lipid distance perimeter. H. Mitochondrial interactions with lipid droplets are calculated by subtracting the mitochondria by the lipid distance contact, then dividing by the percentage of mitochondrial perimeter. I. Mitochondria lipid interface, indicated by the peridroplet mitochondria (PDM), which is equivalent to the percentage of mitochondria in contact with lipid.

Figure S4. Model of dimensions associated with mitochondrial cristae. A. Depiction of crista junction diameter. Cristae are formed by folds in the mitochondrial inner membrane. They are finger-like structures that project out from the cristae where the membrane aligns. The crista junction diameter is the 
length of a crista junction from top to bottom of the crista crypt. The crista crypt starts at the base of the membrane and ends at the tip of the crista junction. B. The intermembrane space is the space between the inner mitochondrial membrane sides. The intermembrane space diameter is the width of a crista. C. The intracristae space width is the distance between two cristae junctions. D. The cristae junction width is the distance between the edges of the same cristae junction. E. Depiction of cristae number calculated by identifying and counting all cristae within a mitochondrion. F. Depiction of cristae surface area (CSA) which starts at cristae junction's base and ends at its tip and wraps around the junction in 3D. The CSA is the average surface area of all cristae in a mitochondrion. G. Depiction of cristae volume density (CVD), a percentage calculated by dividing the CSA by the surface area of the outer mitochondrial membrane $(\mathrm{OMM})$.

Figure S5. Model of cristae ratio and endoplasmic reticulum. A. Depiction of cristae ratio between lamellar and tubular cristae. B. Depiction of endoplasmic reticulum interacting with other organelles. C. Depiction of MERC percentage coverage, calculated by measuring the contact length of the MERC and dividing the surface area of the ER or mitochondria.

Figure S6. Loss of mitochondrial dynamics genes alters the endoplasmic reticulum. TEM micrograph of ER surface area in control and DRP-1 deficient primary skeletal myoblasts A, A'. without and B, B' with color superimposed; mitochondria (dark blue), cristae (light blue), and endoplasmic reticulum (green). C. Surface area in control and DRP-1 deficient primary skeletal myoblasts. TEM micrograph of ER surface area in D, D'. control and E, E'. MFN-2 deficient primary skeletal myoblasts. F. Surface area in control and MFN-2 deficient primary skeletal myoblasts.

Figure S7. Models of electron microscopy and quantification of MAMs. A. Cartoon depiction of proteins associated with MAMs: MFN1, MFN2, SLC25A46, GRP75. Mitochondrial proteins: OPA1 and MICOS; ER proteins: PERK, MFN2, STARD7, EMC, ORP5/8, SIGMAR1, BiP, IP3R3, PACS-2, and VAPB; OMM proteins. VDAC1, DRP1, BAP31, ATAD3A, PTPIP51, and FIS1. The SIGMAR1-BiPIP3R3-GRP75-VDAC1 complex facilitates ER-mitochondria transfer of $\mathrm{Ca}^{2+}$. B. Illustration depicting 
the connection of outer mitochondrial membrane to rough ER (ribo-MERCs) with generic $\mathrm{Ca}^{2+}$ channel and the protein RRBP1 facilitating ER translocation. C. Connection of outer mitochondrial membrane to smooth ER (smooth MERCs). D. MAM distance is the space between the outer membrane of the mitochondria and adjacent ER. E. Mitochondria-endoplasmic reticulum contact sites (MERCs) are depicted as the interaction length along the mitochondria and the ER. F. Depiction of ER-mitochondria contact coefficient (ERMICC). ERMICC computes the MAM distance, MERC length, and mitochondria perimeter involved in the interaction. ERMICC is equal to length divided by perimeter and multiplied by distance.

Figure S8. Measurements associated with cellular recycling machinery. A. The three main types of recycling organelles: lysosomes, autophagosomes, autolysosomes. Lysosomes contain degradative enzymes, which break down cellular debris. Autophagosomes contain cellular material scheduled for autophagy. Lysosomes and autophagosomes often fuse into autolysosomes as they transfer cellular material to be degraded. B. Depiction of omegasome, a subdomain of the ER membrane that consists of lipid bilayer membranes and plays a role in autophagy. The omegasome and ER make up the ERassociated autophagic structure. C. Measurement of lysosomal area. Lysosomal area is related to the diameter of the lysosome, by a factor of $\pi / 2$. D. Lysosome number calculated by dividing the total number of lysosomes in a cell by the area of the cell. E. Autophagosome area is directly related to the diameter of the autophagosome, by a factor of $\pi / 2$. F. Autophagosome number is calculated by dividing the total number of autophagosomes in a cell by the area of the cell. G. Autolysosomal area is directly related to the diameter of the autolysosome, by a factor of $\pi / 2$. H. Autolysosome number is calculated by dividing the total number of autolysosomes in a cell by the area of the cell. I. Depiction of the ERisolation membrane (ER-IM) percentage, which is the percentage of ER-IM in the total autophagic structure and is calculated by dividing the total ER-IM by the total autophagic structure. 